\title{
Modular Symmetries of Threshold Corrections for Abelian Orbifolds with Discrete Wilson Lines
}

\author{
A.Love and S.Todd \\ Royal Holloway and Bedford New College, \\ University of London, \\ Egham, Surrey TW20 OEX. \\ May 1996
}

\begin{abstract}
The modular symmetries of string loop threshold corrections for gauge coupling constants are studied in the presence of discrete Wilson lines for all examples of abelian orbifolds, where the point group is realised by the action of Coxeter elements or generalised Coxeter elements on the root lattices of the Lie groups.
\end{abstract}




\section{Introduction}

Orbifold compactifications of string theory [1, 2] possess various moduli, which are background fields corresponding to marginal deformations of the corresponding conformal field theory, including radii and angles of the underlying six dimensional torus. The spectrum of states for an orbifold is invariant under certain discrete transformations of the moduli, together with the winding numbers and momenta, referred to as modular symmetries [3, 4, 5, 6, 7, 8, 9, 10]. These modular symmetries also appear as symmetries of the string loop threshold corrections [11, 12, 13, 14, 15, 16] which are important for the unification of gauge coupling constants. In this case, it is only the transformations of the moduli associated with fixed planes of twisted sectors of the orbifold theory that are relevant, and it is these particular modular symmetries we shall focus on here. Moreover, the form of the threshold corrections, which is constrained by the modular symmetries, determines the form of the non-perturbative superpotential due to gaugino condensation in the hidden sector and the effective potential, the minimization of which determines the values of the $\mathrm{T}$ and $\mathrm{U}$ moduli [17, 18, 19, 20, 21, 22, 23.

In the absence of Wilson lines, provided all twisted sector fixed planes are such that the six-torus $T^{6}$ can be decomposed as the direct sum $T^{2} \oplus T^{4}$ with the fixed plane lying in $T^{2}$, the group of modular symmetries of the threshold corrections is a product of $\operatorname{PSL}(2, \mathrm{Z})$ factors, one for each of the $\mathrm{T}$ and $\mathrm{U}$ moduli associated with the fixed planes. When such a decomposition is not possible ( the non- $T^{2} \oplus T^{4}$ case) the group of modular symmetries is in general a product of congruence subgroups [24, 25, 26] of $\operatorname{PSL}(2, \mathrm{Z})$. Wilson lines can also break the PSL $(2, \mathrm{Z})$ modular symmetries [27, 28, 29] or further break [30] the congruence subgroups of $\operatorname{PSL}(2, \mathrm{Z})$.

It is our purpose here to classify all possible modular symmetry groups associated with threshold corrections for abelian $\left(Z_{N}\right.$ and $\left.Z_{N} \otimes Z_{M}\right)$ Coxeter and generalised Coxeter orbifolds, for abitrary choices of (discrete) Wilson lines. In section 2, the Coxeter and generalised Coxeter abelian orbifolds are classified. In section 3 , the modular symmetry groups in the presence of Wilson lines are derived in the $T^{2} \oplus T^{4}$ cases, in the sense discussed above, and in section 4 they are derived for the non- $T^{2} \oplus T^{4}$ cases. Section 5 contains our conclusions.

\section{Coxeter and Generalised Coxeter Orbifolds}

A large class of abelian $\left(Z_{N}\right.$ and $\left.Z_{N} \otimes Z_{M}\right)$ orbifolds, the Coxeter orbifolds, can be obtained by taking the underlying lattice of the six-torus to be a 
direct sum of Lie group root lattices and constructing the generators of the point group from Coxeter elements, generalised Coxeter elements or their powers for the various root lattices. A Coxeter element is the product of all the Weyl reflections of the root lattice. When the Dynkin diagram possesses an outer automorphism, we can also make a generalised Coxeter element by using those Weyl reflections associated with points in the Dynkin diagram that are not permuted by the outer automorphism together with one of the permuted Weyl reflections and the outer automorphism itself [31].

The only abelian groups that are able to act as automorphisms of a 6 dimensional lattice and produce exactly $N=1$ supersymmetry in the four dimensional compactified theory [1] are $Z_{N}$ and $Z_{M} \otimes Z_{N}$ discrete subgroups of $\mathrm{SU}(3)$. These groups are given in tables 1 and 2 together with the action of the point group on the 3 complex planes in the space basis (i.e. with the bosonic degrees of freedom for the string on the compact manifold $X^{1} \ldots X^{6}$, written as the combinations $\frac{1}{\sqrt{2}}\left(X^{1}+i X^{2}\right), \frac{1}{\sqrt{2}}\left(X^{3}+i X^{4}\right)$, and $\frac{1}{\sqrt{2}}\left(X^{5}+i X^{6}\right)$.) We need all possible choices of Lie group root lattices and Coxeter or generalised Coxeter elements which are able to realise these point groups. The order $\mathrm{N}$ of the Coxeter element is given by [31,

$$
N=\frac{\text { number of non }- \text { zero roots }}{\text { rank of Lie algebra }}
$$

In the case of a generalised Coxeter element, the rank of the algebra is replaced by an effective rank which is the rank reduced by the order of the outer automorphism minus one. The possible root lattices of dimensions less than or equal to 6 where the Coxeters or generalised Coxeters realise the various relevant $Z_{N}$ point groups are given in table 3 .

Even if the correct $Z_{N}$ point group is realised by Coxeters or generalised Coxeters acting on Lie group root lattices, it is still necessary to check that their eigenvalues correspond to the correct action of the point group element on the complex planes in the space basis. This eliminates many possibilities. All possible choices of lattices for Coxeter and generalised Coxeter $Z_{N}$ orbifolds have already been presented in [31], and are included in table 1 for convenience. For $Z_{N} \times Z_{M}$ orbifolds, some examples have already been given [32, 33]. If we insist on realising the point group using only Coxeters and generalised Coxeters but not their powers, the only possibilities are as in table 2. Some $Z_{N} \times Z_{M}$ point groups cannot be realised at all in this way. Appendix B contains the explicit action of the point group on the lattices for each $Z_{N}$ or $Z_{M} \times Z_{N}$ example. The matrices for the various Coxeter and generalised Coxeter elements involved are given in appendix $\mathrm{A}$.

In the absence of Wilson lines, the modular symmetries of threshold corrections and the threshold corrections themselves have already been calculated 
for all $Z_{N}$ Coxeter orbifolds [24, 25, 26] with non-decomposable twisted sector fixed planes. It remains to carry out the calculations for the generalised Coxeter orbifold $Z_{6}$-II-f with a non $-T_{2} \oplus T_{4} Z_{2}$ fixed plane in the $\theta^{2}$ twisted sector. This is done in appendix C. None of the $Z_{M} \times Z_{N}$ example have non $-T_{2} \oplus T_{4}$ planes.

\section{Effect of Wilson Lines on Modular Symme- tries for Decomposable Fixed Planes}

As discussed in the introduction, it is the orbifold twisted sectors that possess fixed planes that are relevant to threshold corrections to gauge coupling constants. In this section, we shall discuss the modular symmetries associated with these twisted sectors in the presence of Wilson lines for the simplest case where the 6 torus $T^{6}$ can be decomposed as a direct sum $T^{2} \oplus T^{4}$ with the fixed plane lying in the $T^{2}$.

The case of $Z_{N}$ Coxeter or generalised Coxeter orbifolds is discussed first. Let the Wilson line associated with the lattice vector $e_{a}^{i}$ be denoted by $A_{a}^{I}$ where $\mathbf{I}$ is a space index for the $E_{8} \times E_{8}$ degrees of freedom and $\mathbf{a}$ is a lattice index for $T^{6}$. The possible values for the Wilson lines are constrained by the usual [35] homomorphism and worldsheet modular invariance conditions. For a $Z_{N}$ orbifold these are given by,

$$
N A_{a}^{I} \in \Lambda_{E_{8} \times E_{8}}
$$

where $\Lambda_{E_{8} \times E_{8}}$ is the $E_{8} \times E_{8}$ lattice, and

$$
N\left(\sum_{I}\left(n V^{I}+r_{a} A_{a}^{I}\right)^{2}-n^{2} v^{2}\right)=0 \quad(\bmod 2)
$$

where $n=0,1 \ldots N-1, r_{a}=0,1, \ldots N-1, V^{I}$ is the embedding of the point group element $\theta$ in $E_{8} \times E_{8}$ and the action of $\theta$ in the $j$ th complex plane is $e^{2 \pi i v_{j}}, j=1,2,3$.

$$
\begin{array}{r}
N \sum_{I}\left(A_{a}^{I}\right)^{2}=0 \quad(\bmod 2) \\
N \sum_{I} A_{a}^{I} A_{b}^{I}=0 \quad(\bmod 1), a \neq b \\
N \sum_{I} V^{I} A_{a}^{I}=0 \quad(\bmod 1) .
\end{array}
$$

If the action of the point group element $\theta$ on the basis vectors of the compact manifold is

$$
\theta: e_{a} \rightarrow \Theta_{a b} e_{b}
$$


then

$$
A_{a}=\Theta_{a b} A_{b}+\Lambda_{E_{8} \times E_{8}}
$$

Identifies equivalent Wilson lines and contains the homomorphism condition (2). Equivalent Wilson lines are listed in appendix B for the various orbifolds. Moreover, by subtracting off appropriate lattice vectors we can choose lattice vectors in the region

$$
\sum_{I=1}^{9}\left(A_{a}^{I}\right)^{2} \leq 1, \sum_{I=9}^{16}\left(A_{a}^{I}\right)^{2} \leq 1
$$

where the two conditions are seen to apply to the components of the Wilson line in the first and second $E_{8}$ factors of the gauge group separately.

For the purpose of defining modular symmetries it is more convenient to work in terms of the matrix $A_{A b}$ defined by

$$
A_{a B}^{T}=\sum_{I} A_{a}^{I} E_{B}^{I}
$$

where $E_{A}^{I}$ is a basis for the $E_{8} \times E_{8}$ lattice. We can write (10) as

$$
A_{a B}^{T}=A_{a}^{I} X_{I B}^{-1}
$$

where $X$ is the matrix

$$
X=\frac{1}{2}\left(\begin{array}{cccccccc}
2 & -2 & 0 & 0 & 0 & 0 & 0 & 0 \\
0 & 2 & -2 & 0 & 0 & 0 & 0 & 0 \\
0 & 0 & 0 & 2 & -2 & 0 & 0 & 0 \\
0 & 0 & 0 & 0 & 2 & -2 & 0 & 0 \\
0 & 0 & 0 & 0 & 0 & 2 & -2 & 0 \\
0 & 0 & 0 & 0 & 0 & 0 & 2 & 2 \\
-1 & -1 & -1 & -1 & -1 & 1 & 1 & -1
\end{array}\right)
$$

In this formulation [27, 28, 36] acceptable Wilson lines must satisfy

$$
A(I-Q) \in Z
$$

and

$$
\frac{1}{2} A^{T} C A(I-Q)+\frac{1}{2}\left(I-Q^{*}\right) A^{T} C A \in Z
$$

where

$$
Q=\Theta^{T}
$$


and

$$
Q^{*} \equiv\left(Q^{-1}\right)^{T}
$$

These conditions reflect the fact that the action of the point group must be an automorphism of the whole Narain lattice in the presence of Wilson lines [36. Equations (13), (14) and (8) are slightly more restrictive than (4),(5) and (8), and in practice, we find it convenient to generate Wilson lines consistent with (4),(5) and (8) and then retain only those which also satisfy (13) and (14). For a specific choice of embedding of the point group we would also have to satisfy (6).

In the case of a $T^{2} \oplus T^{4}$ fixed plane, the problem of determining the modular symmetries for the moduli associated with this plane reduces to a 2 dimensional problem 29. If $T$ and, in the case of a $Z_{2}$ plane, $\mathrm{U}$, are the moduli associated with this fixed plane, then the full $\operatorname{PSL}(2, \mathrm{Z})$ group of modular transformations may be written as

$$
T \rightarrow \frac{a T+b}{c T+d}, a, b, c, d \in Z, a d-b c=1
$$

and

$$
U \rightarrow \frac{a^{\prime} U+b^{\prime}}{c^{\prime} U+d^{\prime}}, a^{\prime}, b^{\prime}, c^{\prime}, d^{\prime} \in Z, a^{\prime} d^{\prime}-b^{\prime} c^{\prime}=1
$$

If the fixed plane is a $Z_{k}$ fixed plane with $k \neq 2$ i.e. if the action of the point group in that complex plane is $Z_{k}$ with $k \neq 2$, then only the $T$ modulus occurs as a variable parameter of the orbifold model.

In general, the Wilson lines associated with this plane break the PSL $(2, \mathrm{Z})$ modular groups to subgroups. For the $T$ modular transformations, the conditions that determine this subgroup are [29]

$$
\begin{aligned}
c A & \in Z \\
\frac{c}{2} A^{T} C A & \in Z \\
c A J A^{T} C & \in Z \\
(1-d) A-\frac{c}{2} A J A^{T} C A & \in Z \\
(1-a) C A-\frac{c}{2} C A J A^{T} C A & \in Z \\
\left(1-\frac{a}{2}-\frac{d}{2}\right) A^{T} C A-\frac{c}{4} A^{T} C A J A^{T} C A & \in Z
\end{aligned}
$$

where $C$ is the Cartan metric for $E_{8} \times E_{8}$ and

$$
J=\left(\begin{array}{cc}
0 & 1 \\
-1 & 0
\end{array}\right)
$$


For the $\mathrm{U}$ modular transformations, the conditions that determine the unbroken subgroup are

$$
\begin{aligned}
A\left(I-F^{-1}\right) & \in Z \\
A^{T} C A-\frac{1}{2} A^{T} C A F^{-1}-\frac{1}{2} F^{T} A^{T} C A & \in Z
\end{aligned}
$$

where

$$
F=\left(\begin{array}{ll}
d^{\prime} & b^{\prime} \\
c^{\prime} & a^{\prime}
\end{array}\right)
$$

In (19)-(27) the fixed plane has been treated as an independent 2 dimensional plane and $A$ and $A^{T}$ have been truncated to the relevant two columns and rows respectively, which correspond to the fixed plane.

We have determined all possible modular symmetry groups in the presence of Wilson lines for the $T_{2} \oplus T_{4}$ fixed planes of $Z_{N}$ Coxeter and generalised Coxeter orbifolds. These are given in appendix D along with examples of Wilson lines that break the full PSL $(2, \mathrm{Z})$ group to these subgroups. For the case of a $T_{2} \oplus T_{4}$ plane the resulting modular groups do not depend directly on the specific $Z_{N}$ orbifold, but only on the action of the point group in that fixed plane. However, if there are Wilson lines present in other complex planes they can affect the allowed values of the Wilson lines in the fixed plane through the world sheet modular invariance conditions or (14), because the Cartan matrix $C$ links rows of $A^{T}$ corresponding to different complex planes. In that case, the full action of the point group is of importance because of its influence on inequivalent Wilson lines in the other complex planes. We have presented all modular groups permitted for the fixed plane with nonzero Wilson lines only in the fixed plane. With non-zero Wilson lines also present in other complex planes the possibilities are reduced because of the restriction of the choices of Wilson lines in the fixed plane just discussed. Specific choices of the point group embedding will further limit the choices of Wilson lines and so the possible modular groups.

For the $Z_{M} \times Z_{N}$ orbifolds, the allowed Wilson lines are determined by equivalence conditions associated with both point group elemnents $\theta$ and $\omega$ (as listed in appendix B) and by the worldsheet modular invariance conditions [35] defined for the $\theta^{k} \omega^{l}$ twisted sector

$$
N^{\prime}\left(\sum_{I}\left(k A^{I}+l B^{I}+r_{a} A_{a}^{I}\right)^{2}-(k a+l b)^{2}\right)=0 \quad(\bmod 2)
$$

where $k=0,1, \ldots, M-1, l=0,1, \ldots, N-1, N^{\prime}$ is the order of $\theta^{k} \omega^{l}, A^{I}$ and $B^{I}$ are the embeddings of $\theta$ and $\omega$ in the $E_{8} \times E_{8}$ and the action of $\theta$ 
and $\omega$ on the $j$ th complex plane is $e^{2 \pi i a_{j}}, j=1,2,3$, and $e^{2 \pi i b_{i}}, i=1,2,3$. The integers $r_{a}$ take values $0,1, \ldots, N^{\prime}-1$.

In general, there could be two sets of conditions of the type (13) and (14) to satisfy, one for $Q(\theta)$ and one for $Q(\omega)$. However, by inspection of appendix B we see that for the most part, only one of $Q(\theta)=\Theta^{T} Q(\omega)=\Omega^{T}$ acts non-trivially in a particular fixed plane, or $Q(\theta)$ and $Q(\omega)$ both act in the same way. The only cases where $Q(\theta)$ and $Q(\omega)$ act differently are when one acts as $C^{T}(S U(3))$ and the other acts as $C^{T}\left(S U(3)^{[2]}\right)$. However, in these cases there is no breaking of the $\operatorname{PSL}(2, \mathrm{Z})$ modular groups. The resulting modular symmetry groups in the presence of Wilson lines in the fixed plane are given in appendix E.

\section{Effect of Wilson Lines on Modular Symme- tries for non- $T_{2} \oplus T_{4}$ Fixed Planes}

When a fixed plane in an orbifold twisted sector is such that the 6 torus $T^{6}$ can not be decomposed into a direct sum $T^{2} \oplus T^{4}$ with the fixed plane lying in the $T^{2}$, the associated modular symmetry groups for $T$ and $U$ moduli are, in general, already broken to subgroups of $\operatorname{PSL}(2, \mathrm{Z})$ even in the absence of Wilson lines [24, 25, 26]. In the presence of Wilson lines further breaking of the symmetry can occur. A systematic method of calculating the resulting modular symmetry groups has been given elsewhere [30]. The system of equations that determine these modular groups depends on two matrices [38], which we call $\mathrm{R}$ and $\mathrm{K}$. The role of these two matrices is that of allowing the initially 6 dimensional problem to be reduced to a 2 dimensional form. In appendix $\mathrm{E}$ we list all the $\mathrm{R}$ and $\mathrm{K}$ matrices for the models considered and in appendix $\mathrm{F}$ we explain the derivation of these matrices and thus how they relate to the notation in previous papers ([30] and [33]). There are no $Z_{M} \times Z_{N}$ orbifolds with point groups elements realised by Coxeter or generalised Coxeter elements with non- $T_{2} \oplus T_{4}$ fixed planes, with the exeception of $Z_{2} \times Z_{2}$ which was considered in [37].

To solve for the modular symmetries, it is convenient to construct an $8 \times 2$ matrix, which we write simply as $A_{T}$ in the following, which contains the relevant Wilson line components involved in the problem and is obtained in the reduction from the 6 dimensional problem, as detailed in appendix F. The modular symmetries, then, are given by the constraint equations, which are for the $\mathrm{T}$ modulus

$$
\begin{array}{r}
c A_{T} R J K \in Z \\
c A_{T} R J K A_{T}^{t} C \in Z
\end{array}
$$




$$
\begin{aligned}
(1-d) A_{T} R-\frac{1}{2} c A_{T} R J K A_{T}^{t} C A_{T} R & \in Z \\
c K A_{T}^{t} C & \in Z \\
\frac{1}{2} c K A_{T}^{t} C A_{T} R & \in Z \\
\frac{1}{2} c A_{T}^{t} C A_{T} R J K & \in Z \\
\left(1-\frac{1}{2} a-\frac{1}{2} d\right) A_{T}^{t} C A_{T} R-\frac{1}{4} c A_{T}^{t} C A_{T} R J K A_{T}^{t} C A_{T} R+b K^{-1} J & \in Z
\end{aligned}
$$

and for the U modulus

$$
\begin{aligned}
A_{T} R(I-M) & \in Z \\
K^{-1} M^{*} K & \in Z \\
A_{T}^{t} C A_{T} R-\frac{1}{2} A_{T}^{t} C A_{T} R M-\frac{1}{2} K^{-1} M^{*} K A_{T}^{t} C A_{T} R & \in Z
\end{aligned}
$$

where $\mathrm{J}$ is given by (25)

$$
M=\left(\begin{array}{cc}
a^{\prime} & -b^{\prime} \\
-c^{\prime} & d^{\prime}
\end{array}\right)=F^{-1}
$$

and $M^{*}$ is defined to be $\left(M^{-1}\right)^{t}$. The integer coefficients $a, b, c, d$ and $a^{\prime}, b^{\prime}, c^{\prime}, d^{\prime}$ in the constraint equations are the coefficients of the modular transformations (17,18) on the $T$ and $U$ moduli respectively.

\section{Conclusions}

The moduli dependent corrections to gauge coupling constants in orbifold compactifactions are a possible mechanism for reconciling the unification scale for the standard model gauge coupling constants with string theory. We have carried out a systematic study of the possible modular symmetry groups in the presence of quantised Wilson lines for all Coxeter orbifolds. Modular symmetry constrains the functional form that moduli dependent threshold corrections can take and, in some cases, this constraint is strong enough to explicitly determine the threshold correction. The results of the present paper are a first step in that direction for all Coxeter orbifolds. The modular symmetry groups obtained are in some cases congruence subgroups and in other cases more exotic subgroups of $\operatorname{PSL}(2, \mathrm{Z})$. 


\section{Appendices}

\section{A Coxeter and Generalised Coxeter Elements}

For each Lie group root lattice the action of the Coxeter element or generalised Coxeter element on the basis vectors $e_{a}$ of the root lattice is given below. The action of the Weyl reflection $\sigma_{a}$ associated with the basis vector $e_{a}$ on the basis vector $e_{b}$ is defined to be

$$
\sigma_{a}\left(e_{b}\right)=e_{b}-\frac{2 e_{a} \cdot e_{b}}{e_{a} \cdot e_{a}} e_{a}
$$

The generalised Coxeter elements also contain cyclic permutations of the basis vectors

\section{A.1 $\quad \mathrm{SU}(2)$}

Dynkin diagram $\bigcirc^{1}$

Coxeter $\theta=\sigma_{1}$

$\theta e_{1}=-e_{1}$

\section{A.2 SU(3)}

Dynkin diagram

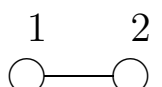

Coxeter $\theta=\sigma_{1} \sigma_{2}$

$\theta e_{1}=e_{2}, \theta e_{2}=-e_{1}-e_{2}$

Eigenvalues $\exp ( \pm 2 \pi i / 3)$

Generalised Coxeter $\theta=\sigma_{2}\left(\begin{array}{ll}1 & 2 \\ 2 & 1\end{array}\right)$

$\theta e_{1}=-e_{2}, \theta e_{2}=e_{1}+e_{2}$

Eigenvalues $\exp ( \pm 2 \pi i / 6)$

\section{A.3 SU(4)}

Dynkin diagram $\bigcirc$

Coxeter $\theta=\sigma_{1} \sigma_{2} \sigma_{3}$

$\theta e_{1}=e_{2}, \theta e_{2}=e_{3}, \theta e_{3}=-e_{1}-e_{2}-e_{3}$

Eigenvalues $-1, \pm i$

Generalised Coxeter $\theta=\sigma_{1} \sigma_{2}\left(\begin{array}{ll}1 & 3 \\ 3 & 1\end{array}\right)$ 


\begin{tabular}{|l|c|c|}
\hline Orbifold & $\theta$ & Lattice \\
\hline$Z_{3}$ & $(1,1,-2) / 3$ & $S U(3) \times S U(3) \times S U(3)$ \\
$Z_{4}-a$ & $(1,1,-2) / 4$ & $S U(4) \times S U(4)$ \\
$Z_{4}-b$ & $(1,1,-2) / 4$ & $S U(4) \times S O(5) \times S U(2)$ \\
$Z_{4}-c$ & $(1,1,-2) / 4$ & $S O(5) \times S U(2) \times S O(5) \times S U(2)$ \\
$Z_{6}--I--a$ & $(-2,1,1) / 6$ & $S U(3) \times G_{2} \times G_{2}$ \\
$Z_{6}--I--b$ & $(-2,1,1) / 6$ & $S U(3) \times S U(3)^{[2]} \times S U(3)^{[2]}$ \\
$Z_{6}-I-c$ & $(-2,1,1) / 6$ & $S U(3) \times S U(3)^{[2]} \times G_{2}$ \\
$Z_{6}-I I-a$ & $(2,1,-3) / 6$ & $S U(6) \times S U(2)$ \\
$Z_{6}-I I-b$ & $(2,1,-3) / 6$ & $S U(3) \times S O(8)$ \\
$Z_{6}-I I-c$ & $(2,1,-3) / 6$ & $S U(3) \times S O(7) \times S U(2)$ \\
$Z_{6}-I I-d$ & $(2,1,-3) / 6$ & $S U(3) \times G_{2} \times S O(4)$ \\
$Z_{6}-I I-e$ & $(2,1,-3) / 6$ & $S U(3) \times S U(3)^{[2]} \times S O(4)$ \\
$Z_{6}-I I-f$ & $(2,1,-3) / 6$ & $S U(3) \times S U(4)^{[2]} \times S U(2)$ \\
$Z_{6}-I I-g$ & $(2,1,-3) / 6$ & $S U(3) \times S p(6) \times S U(2)$ \\
$Z_{7}$ & $(1,2,-3) / 7$ & $S U(7)$ \\
$Z_{8}-I-a$ & $(1,-3,2) / 8$ & $S O(9) \times S O(5)$ \\
$Z_{8}-I-b$ & $(1,-3,2) / 8$ & $S O(8)^{[2]} \times S O(5)$ \\
$Z_{8}-I I-a$ & $(1,3,-4) / 8$ & $S O(10) \times S U(2)$ \\
$Z_{8}-I I-b$ & $(1,3,-4) / 8$ & $S O(9) \times S O(4)$ \\
$Z_{8}-I I-c$ & $(1,3,-4) / 8$ & $S O(8)^{[2]} \times S O(4)$ \\
$Z_{12}-I-a$ & $(1,-5,4) / 12$ & $E_{6}$ \\
$Z_{12}-I-b$ & $(1,-5,4) / 12$ & $F_{4} \times S U(3)$ \\
$Z_{12}-I-c$ & $(1,-5,4) / 12$ & $S O(8)^{[3]} \times S U(3)$ \\
$Z_{12}-I I-a$ & $(1,5,-6) / 12$ & $F_{4} \times S O(4)$ \\
$Z_{12}-I I-b$ & $(1,5,-6) / 12$ & $S O(8)^{[3]} \times S O(4)$ \\
\hline
\end{tabular}

Table 1: Coxeter and generalised Coxeter $Z_{N}$ orbifolds. For the point group generator $\theta$ we display $\left(v_{1}, v_{2}, v_{3}\right)$ such that the action of $\theta$ in the complex orthogonal basis is $\left(e^{2 \pi i v_{1}}, e^{2 \pi i v_{2}}, e^{2 \pi i v_{2}}\right)$. When the generalised Coxeter element with outer automorphism of order $p$ acts on the root lattice is written as $G^{[p]}$. 


\begin{tabular}{|l|c|c|c|}
\hline Orbifold & $\theta$ & $\omega$ & Lattice \\
\hline$Z_{2} \times Z_{2}$ & $(1,0,-1) / 2$ & $(0,1,-1) / 2$ & $(S O(4))^{3}$ \\
$Z_{3} \times Z_{3}$ & $(1,0,-1) / 3$ & $(0,1,-1) / 3$ & $(S U(3))^{3}$ \\
$Z_{2} \times Z_{4}$ & $(1,0,-1) / 2$ & $(0,1,-1) / 4$ & - \\
$Z_{4} \times Z_{4}$ & $(1,0,-1) / 4$ & $(0,1,-1) / 4$ & $(S O(5))^{3}$ \\
$Z_{2} \times Z_{6}$ & $(1,0,-1) / 2$ & $(0,1,-1) / 6$ & - \\
$Z_{2} \times Z_{6}^{\prime}$ & $(1,0,-1) / 2$ & $(0,1,-1) / 6$ & - \\
$Z_{3} \times Z_{6}-a$ & $(1,0,-1) / 3$ & $(0,1,-1) / 6$ & $(S U(3))^{3}$ \\
$Z_{3} \times Z_{6}-b$ & $(1,0,-1) / 3$ & $(0,1,-1) / 6$ & $S U(3) \times G_{2} \times S U(3)$ \\
$Z_{6} \times Z_{6}-a$ & $(1,0,-1) / 6$ & $(0,1,-1) / 6$ & $\left(G_{2}\right)^{3}$ \\
$Z_{6} \times Z_{6}-b$ & $(1,0,-1) / 6$ & $(0,1,-1) / 6$ & $\left(G_{2}\right)^{2} \times S U(3)$ \\
$Z_{6} \times Z_{6}-c$ & $(1,0,-1) / 6$ & $(0,1,-1) / 6$ & $G_{2} \times(S U(3))^{2}$ \\
$Z_{6} \times Z_{6}-d$ & $(1,0,-1) / 6$ & $(0,1,-1) / 6$ & $(S U(3))^{3}$ \\
\hline
\end{tabular}

Table 2: Coxeter and generalised Coxeter $Z_{M} \times Z_{N}$ orbifolds. For the point group generator $\theta$ we display $\left(v_{1}, v_{2}, v_{3}\right)$ such that the action of $\theta$ in the complex orthogonal basis is $\left(e^{2 \pi i v_{1}}, e^{2 \pi i v_{2}}, e^{2 \pi i v_{2}}\right)$ and similarly for $\omega$.

\begin{tabular}{|c|c|}
\hline Point Group & Possible Root Lattices \\
\hline$Z_{2}$ & $S U(2), S O(4)$ \\
$Z_{3}$ & $S U(3)$ \\
$Z_{4}$ & $S U(4), S O(5)$ \\
$Z_{6}$ & $S U(6), S O(8), S O(7), S p(6)$, \\
& $G_{2}, S U(3)^{[2]}, S U(4)^{[2]}$ \\
$Z_{7}$ & $S U(7)$ \\
$Z_{8}$ & $S O(10), S O(9), S p(8), S O(8)^{[2]}$ \\
$Z_{12}$ & $S O(13), S p(12), F_{4}, E_{6}, S O(8)^{[3]}$ \\
\hline
\end{tabular}

Table 3: Root lattices of dimension $\leq 6$ where the Coxeter or generalised Coxeter element generate $Z_{N}$ point groups. The generalised Coxeter elements with outer automorphism of order $p$ acintg on the root lattice is written as $G^{[p]}$. 
$\theta e_{1}=e_{1}+e_{2}+e_{3}, \theta e_{2}=-e_{1}-e_{2}, \theta e_{3}=e_{2}$

Eigenvalues $-1, \exp ( \pm 2 \pi i / 6)$

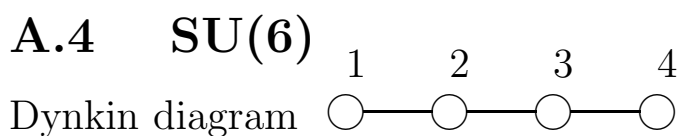

Coxeter $\theta=\sigma_{1} \sigma_{2} \sigma_{3} \sigma_{4} \sigma_{5}$

$\theta e_{1}=e_{2}, \theta e_{2}=e_{3}, \theta e_{3}=e_{4}, \theta e_{4}=e_{5}, \theta e_{5}=-e_{1}-e_{2}-e_{3}-e_{4}-e_{5}$

Eigenvalues $-1, \exp ( \pm 2 \pi i / 3), \exp ( \pm 2 \pi i / 6)$

\section{A.5 SU(7)}

Dynkin diagram

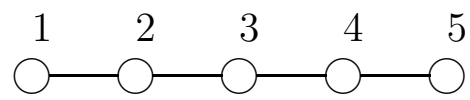

Coxeter $\theta=\sigma_{1} \sigma_{2} \sigma_{3} \sigma_{4} \sigma_{5} \sigma_{6}$

$\theta e_{1}=e_{2}, \theta e_{2}=e_{3}, \theta e_{3}=e_{4}, \theta e_{4}=e_{5}, \theta e_{5}=e_{6}$,

$\theta e_{6}=-e_{1}-e_{2}-e_{3}-e_{4}-e_{5}-e_{6}$

Eigenvalues $\exp ( \pm 2 \pi i / 7), \exp ( \pm 4 \pi i / 7), \exp ( \pm 6 \pi i / 7)$

\section{A.6 SO(5)}

Dynkin diagram

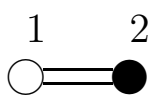

Coxeter $\theta=\sigma_{1} \sigma_{2}$

$\theta e_{1}=e_{1}+2 e_{2}, \theta e_{2}=-e_{1}-e_{2}$

Eigenvalues $\pm i$

\section{A.7 $\quad \mathrm{SO}(7)$}

Dynkin diagram

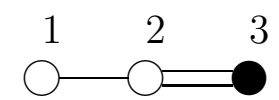

Coxeter $\theta=\sigma_{1} \sigma_{2} \sigma_{3}$

$\theta e_{1}=e_{2}, \theta e_{2}=e_{1}+e_{2}+2 e_{3}, \theta e_{3}=-e_{1}-e_{2}-e_{3}$

Eigenvalues $-1, \exp ( \pm 2 \pi i / 6)$

\section{A.8 $\mathrm{SO}(8)$}

Dynkin diagram

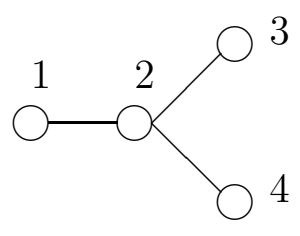


Coxeter $\theta=\sigma_{1} \sigma_{2} \sigma_{3} \sigma_{4}$

$\theta e_{1}=e_{2}, \theta e_{2}=e_{1}+e_{2}+e_{3}+e_{4}, \theta e_{3}=-e_{1}-e_{2}-e_{3}, \theta e_{4}=-e_{1}-e_{2}-e_{4}$

Eigenvalues -1 (twice), $\exp ( \pm 2 \pi i / 6)$

First generalised Coxeter $\theta=\sigma_{1} \sigma_{2} \sigma_{3}\left(\begin{array}{ll}3 & 4 \\ 4 & 3\end{array}\right)$

$\theta e_{1}=e_{2}, \theta e_{2}=e_{3}, \theta e_{3}=e_{1}+e_{2}+e_{4}, \theta e_{4}=-e_{1}-e_{2}-e_{3}$,

Eigenvalues $\exp ( \pm 2 \pi i / 8), \exp ( \pm 10 \pi i / 8)$

Second generalised Coxeter $\theta=\sigma_{1} \sigma_{2}\left(\begin{array}{lll}1 & 3 & 4 \\ 3 & 4 & 1\end{array}\right)$

$\theta e_{1}=e_{1}+e_{2}+e_{3}, \theta e_{2}=-e_{1}-e_{2}, \theta e_{3}=e_{1}+e_{2}+e_{4}, \theta e_{4}=e_{2}$

Eigenvalues $\exp ( \pm 2 \pi i / 12), \exp ( \pm 7 \pi i / 6)$

\section{A.9 $\quad \mathrm{SO}(9)$}

Dynkin diagram

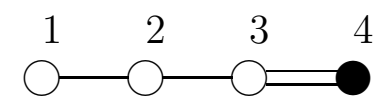

Coxeter $\theta=\sigma_{1} \sigma_{2} \sigma_{3} \sigma_{4}$

$\theta e_{1}=e_{2}, \theta e_{2}=e_{3}, \theta e_{3}=e_{1}+e_{2}+e_{3}+2 e_{4}, \theta e_{4}=-e_{1}-e_{2}-e_{3}-e_{4}$

Eigenvalues $\exp ( \pm 2 \pi i / 8), \exp ( \pm 6 \pi i / 8)$

\section{A.10 SO $(10)$}

Dynkin diagram

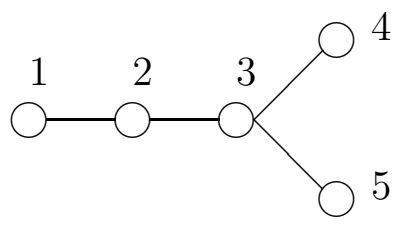

Coxeter $\theta=\sigma_{1} \sigma_{2} \sigma_{3} \sigma_{4} \sigma_{5}$

$\theta e_{1}=e_{2}, \theta e_{2}=e_{3}, \theta e_{3}=e_{1}+e_{2}+e_{3}+e_{4}+e_{5}, \theta e_{4}=-e_{1}-e_{2}-e_{3}-e_{4}$,

$\theta e_{5}=-e_{1}-e_{2}-e_{3}-e_{5}$

Eigenvalues $-1, \exp ( \pm 2 \pi i / 8), \exp ( \pm 6 \pi i / 8)$

\section{A.11 $G_{2}$}

Dynkin diagram

Coxeter $\theta=\sigma_{1} \sigma_{2}$

$\theta e_{1}=e_{1}+2 e_{2}, \theta e_{2}=-e_{1}-e_{2}$

Eigenvalues $\pm i$
A.12 $F_{4}$
Dynkin diagram

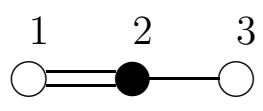


Coxeter $\theta=\sigma_{1} \sigma_{2} \sigma_{3} \sigma_{4}$

$\theta e_{1}=e_{2}, \theta e_{2}=e_{1}+e_{2}+e_{3}, \theta e_{3}=e_{4}, \theta e_{4}=-2 e_{1}-2 e_{2}-e_{3}-e_{4}$

Eigenvalues $\exp ( \pm 2 \pi i / 12), \exp ( \pm 10 \pi i / 12)$

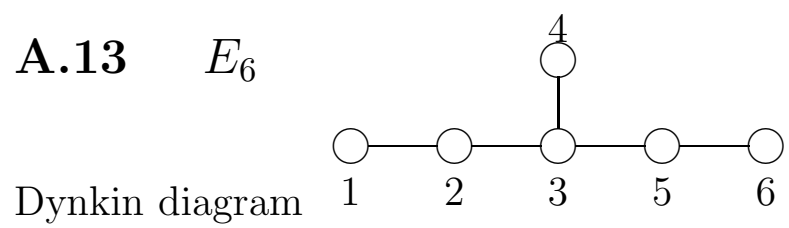

Coxeter $\theta=\sigma_{1} \sigma_{2} \sigma_{3} \sigma_{4} \sigma_{5} \sigma_{6}$

$\theta e_{1}=e_{2}, \theta e_{2}=e_{3}, \theta e_{3}=e_{1}+e_{2}+e_{3}+e_{4}+e_{5}, \theta e_{4}=-e_{1}-e_{2}-e_{3}-e_{4}$,

$\theta e_{5}=e_{6}, \theta e_{6}=-e_{1}-e_{2}-e_{3}-e_{5}-e_{6}$,

Eigenvalues $\exp ( \pm 2 \pi i / 3), \exp ( \pm 2 \pi i / 12), \exp ( \pm 10 \pi i / 12)$

\section{A.14 Sp(6)}

Dynkin diagram

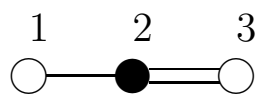

Coxeter $\theta=\sigma_{1} \sigma_{2} \sigma_{3}$

$\theta e_{1}=e_{2}, \theta e_{2}=e_{1}+e_{2}+e_{3}, \theta e_{3}=-2 e_{1}-2 e_{2}-e_{3}$

Eigenvalues $-1, \exp ( \pm 2 \pi i / 6)$

\section{A.15 $\operatorname{Sp}(8)$}

Dynkin diagram

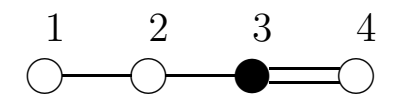

Coxeter $\theta=\sigma_{1} \sigma_{2} \sigma_{3} \sigma_{4}$

$\theta e_{1}=e_{2}, \theta e_{2}=e_{3}, \theta e_{3}=e_{1}+e_{2}+e_{3}+e_{4}, \theta e_{4}=-2 e_{1}-2 e_{2}-2 e_{3}-e_{4}$

Eigenvalues $\exp ( \pm 2 \pi i / 8), \exp ( \pm 6 \pi i / 8)$

\section{B Coxeter and Generalised Coxeter Orbifolds}

For each orbifold, the action of the point group on the complex space basis $\exp \left(2 \pi i\left[v_{1}, v_{2}, v_{3}\right]\right)$ is specified by $\left(v_{1}, v_{2}, v_{3}\right)$. The action of the point group on the lattice basis is given in terms of Coxeter elements or generalised Coxeter elements for the root lattices involved, with the Coxeter element for the root lattice of the Lie group $G$ written as $C(G)$ and any generalised Coxeter element written as $C\left(G^{[p]}\right)$ where $p$ is the order of the outer automorphism involved. Then the action in the lattice basis of the point group element $\theta$ is given in terms of a matrix $\Theta$ composed of Coxeter element matrices, such that the effect of $\theta$ on the basis vectors $e_{a}$ of the lattice is $e_{a} \rightarrow \Theta_{a b} e_{b}$. The order of the point group for the various $T_{2} \oplus T_{4}$ and non- $T_{2} \oplus T_{4}$ twsited 
sector fixed planes (in the sense of the introduction) is given. Finally, the equivalent Wilson line $A_{i}$ corresponds to the basis vector $e_{i}$.

$$
\begin{aligned}
& \text { B.1 } Z_{3} \quad \theta=(1,1,-2) / 3 \quad(S U(3))^{3} \\
& \Theta=[C(S U(3)), C(S U(3)), C(S U(3)] \\
& \text { no fixed planes. } \\
& A_{2} \approx A_{1}, A_{4} \approx A_{3}, A_{6} \approx A_{5}, 3 A_{1} \approx 3 A_{2} \approx 3 A_{5} \approx 0 \\
& \text { B.2 } \quad Z_{4}-a \quad \theta=(1,1,-2) / 4 \quad(S U(4))^{2} \\
& \Theta=[C(S U(4)), C(S U(4))] \\
& \text { non- } T_{2} \oplus T_{4} Z_{2} \text { fixed plane in the } \theta^{2} \text { twisted sector. } \\
& A_{3} \approx A_{2} \approx A_{1}, A_{6} \approx A_{5} \approx A_{4}, 4 A_{1} \approx 4 A_{4} \approx 0
\end{aligned}
$$$$
\text { B.3 } Z_{4}-b \quad \theta=(1,1,-2) / 4 \quad S U(4) \times S O(5) \times S U(2)
$$$$
\Theta=[C(S U(4)), C(S O(5)), C(S U(2))]
$$$$
\text { non }-T_{2} \oplus T_{4} Z_{2} \text { fixed plane in the } \theta^{2} \text { twisted sector. }
$$$$
A_{3} \approx A_{2} \approx A_{1}, 4 A_{1} \approx A_{4} \approx 2 A_{5} \approx 2 A_{6} \approx 0
$$

$$
\begin{aligned}
& \text { B.4 } Z_{4}-c \quad \theta=(1,1,-2) / 4 \quad S O(5) \times S U(2) \times S O(5) \times S U(2) \\
& \Theta=[C(S O(5)), C(S U(2)), C(S O(5)), C(S U(2))] \\
& T_{2} \oplus T_{4} Z_{2} \text { fixed plane in the } \theta^{2} \text { twisted sector. } \\
& A_{1} \approx A_{4} \approx 2 A_{2} \approx 2 A_{3} \approx 2 A_{5} \approx 2 A_{6} \approx 0
\end{aligned}
$$

$$
\text { B.5 } Z_{6}-I-a \quad \theta=(-2,1,1) / 6 \quad S U(3) \times G_{2} \times G_{2}
$$

$\Theta=\left[C(S U(3)), C\left(G_{2}\right), C\left(G_{2}\right)\right]$

$T_{2} \oplus T_{4} Z_{3}$ fixed plane in the $\theta^{3}$ twisted sector.

$A_{2} \approx A_{1}, 3 A_{1} \approx A_{3} \approx A_{4} \approx A_{5} \approx A_{6} \approx 0$

$$
\begin{aligned}
& \text { B.6 } Z_{6}-I-b \quad \theta=(-2,1,1) / 6 \quad S U(3) \times S U(3)^{[2]} \times S U(3)^{[2]} \\
& \Theta=\left[C(S U(3)), C\left(S U(3)^{[2]}\right), C\left(S U(3)^{[2]}\right)\right] \\
& T_{2} \oplus T_{4} Z_{3} \text { fixed plane in the } \theta^{3} \text { twisted sector. } \\
& A_{2} \approx A_{1}, 3 A_{1} \approx A_{3} \approx A_{4} \approx A_{5} \approx A_{6} \approx 0
\end{aligned}
$$


B.7 $Z_{6}-I-c \quad \theta=(-2,1,1) / 6 \quad S U(3) \times S U(3)^{[2]} \times G_{2}$

$\Theta=\left[C(S U(3)), C\left(S U(3)^{[2]}\right), C\left(G_{2}\right)\right]$

$T_{2} \oplus T_{4} Z_{3}$ fixed plane in the $\theta^{3}$ twisted sector.

$A_{2} \approx A_{1}, 3 A_{1} \approx A_{3} \approx A_{4} \approx A_{5} \approx A_{6} \approx 0$

B.8 $Z_{6}-I I-a \quad \theta=(2,1,-3) / 6 \quad S U(6) \times S U(2)$

$\Theta=[C(S U(6)), C(S U(2))]$

non- $T_{2} \oplus T_{4} Z_{3}$ fixed plane in the $\theta^{3}$ twisted sector.

non- $T_{2} \oplus T_{4} Z_{2}$ fixed plane in the $\theta^{2}$ twisted sector.

$6 A_{1} \approx 2 A_{6} \approx 0, A_{5} \approx A_{4} \approx A_{3} \approx A_{2} \approx A_{1}$

B.9 $Z_{6}-I I-b \quad \theta=(2,1,-3) / 6 \quad S U(3) \times S O(8)$

$\Theta=[C(S U(3)), C(S O(8))]$

$T_{2} \oplus T_{4} Z_{3}$ fixed plane in the $\theta^{3}$ twisted sector.

non- $T_{2} \oplus T_{4} Z_{2}$ fixed plane in the $\theta^{2}$ twisted sector.

$A_{2} \approx A_{1}, 3 A_{1} \approx 0, A_{3} \approx A_{4}, 2 A_{3} \approx 2 A_{5} \approx 2 A_{6} \approx 0, A_{3} \approx-A_{5}-A_{6}$

B.10 $Z_{6}-I I-c \quad \theta=(2,1,-3) / 6 \quad S U(3) \times S O(7) \times S U(2)$

$\Theta=[C(S U(3)), C(S O(7)), C(S U(2))]$

$T_{2} \oplus T_{4} Z_{3}$ fixed plane in the $\theta^{3}$ twisted sector.

non- $T_{2} \oplus T_{4} Z_{2}$ fixed plane in the $\theta^{2}$ twisted sector.

$A_{2} \approx A_{1}, 3 A_{1} \approx A_{3} \approx A_{4} \approx 2 A_{5} \approx 2 A_{6} \approx 0$

B.11 $Z_{6}-I I-d \quad \theta=(2,1,-3) / 6 \quad S U(3) \times G_{2} \times S O(4)$

$\Theta=\left[C(S U(3)), C\left(G_{2}\right), C(S O(4))\right]$

$T_{2} \oplus T_{4} Z_{3}$ fixed plane in the $\theta^{3}$ twisted sector.

$T_{2} \oplus T_{4} Z_{2}$ fixed plane in the $\theta^{2}$ twisted sector.

$A_{2} \approx A_{1}, 3 A_{1} \approx A_{3} \approx A_{4} \approx 2 A_{5} \approx 2 A_{6} \approx 0$

B.12 $Z_{6}-I I-e \quad \theta=(2,1,-3) / 6 \quad S U(3) \times S U(3)^{[2]} \times S O(4)$

$\Theta=\left[C(S U(3)), C\left(S U(3)^{[2]}\right), C(S O(4))\right]$

$T_{2} \oplus T_{4} Z_{3}$ fixed plane in the $\theta^{3}$ twisted sector.

$T_{2} \oplus T_{4} Z_{2}$ fixed plane in the $\theta^{2}$ twisted sector.

$A_{2} \approx A_{1}, 3 A_{1} \approx A_{3} \approx A_{4} \approx 2 A_{5} \approx 2 A_{6} \approx 0$ 


$$
\begin{aligned}
& \text { B.13 } Z_{6}-I I-f \quad \theta=(2,1,-3) / 6 \quad S U(3) \times S U(4)^{[2]} \times S U(2) \\
& \Theta=\left[C(S U(3)), C\left(S U(4)^{[2]}\right), C(S U(2))\right] \\
& T_{2} \oplus T_{4} Z_{3} \text { fixed plane in the } \theta^{3} \text { twisted sector. } \\
& T_{2} \oplus T_{4} Z_{2} \text { fixed plane in the } \theta^{2} \text { twisted sector. } \\
& A_{2} \approx A_{1}, 3 A_{1} \approx A_{3} \approx 0, A_{4} \approx A_{5}, 2 A_{4} \approx 2 A_{6} \approx 0
\end{aligned}
$$$$
\begin{aligned}
& \text { B.14 } Z_{6}-I I-g \quad \theta=(2,1,-3) / 6 \quad S U(3) \times S p(6) \times S U(2) \\
& \Theta=[C(S U(3)), C(S p(6)), C(S U(2))] \\
& T_{2} \oplus T_{4} Z_{3} \text { fixed plane in the } \theta^{3} \text { twisted sector. } \\
& \text { non- } T_{2} \oplus T_{4} Z_{2} \text { fixed plane in the } \theta^{2} \text { twisted sector. } \\
& A_{2} \approx A_{1}, 3 A_{1} \approx 2 A_{3} \approx 0, A_{4} \approx A_{5}, 2 A_{4} \approx 2 A_{6} \approx 0
\end{aligned}
$$$$
\text { B.15 } Z_{7} \quad \theta=(1,2,-3) / 7 \quad S U(7)
$$$$
\Theta=[C(S U(7))]
$$$$
\text { no fixed planes }
$$$$
A_{6} \approx A_{5} \approx A_{4} \approx A_{3} \approx A_{2} \approx A_{1}, 7 A_{1} \approx 0
$$$$
\text { B.16 } Z_{8}-I-a \quad \theta=(1,-3,2) / 8 \quad S O(9) \times S O(5)
$$$$
\Theta=[C(S O(9)), C(S O(5))]
$$$$
T_{2} \oplus T_{4} Z_{4} \text { fixed plane in the } \theta^{4} \text { twisted sector. }
$$$$
A_{1} \approx A_{2} \approx A_{3} \approx A_{5} \approx 2 A_{4} \approx 2 A_{6} \approx 0
$$$$
\text { B.17 } Z_{8}-I-b \quad \theta=(1,-3,2) / 8 \quad S O(8)^{[2]} \times S O(5)
$$$$
\Theta=\left[C\left(S O(8)^{[2]}\right), C(S O(5))\right]
$$$$
T_{2} \oplus T_{4} Z_{4} \text { fixed plane in the } \theta^{4} \text { twisted sector. }
$$$$
A_{4} \approx A_{3} \approx A_{2} \approx A_{1}, 2 A_{1} \approx A_{5} \approx 2 A_{6} \approx 0
$$

B.18 $Z_{8}-I I-a \quad \theta=(1,3,-4) / 8 \quad S O(10) \times S U(2)$

$\Theta=[C(S O(10)), C(S U(2))]$

non- $T_{2} \oplus T_{4} Z_{2}$ fixed plane in the $\theta^{2}$ twisted sector.

$A_{1} \approx A_{2} \approx A_{3}, A_{4} \approx-A_{5}, 2 A_{4} \approx A_{3}, 2 A_{3} \approx 2 A_{6} \approx 0$ 


$$
\begin{aligned}
& \text { B.19 } Z_{8}-I I-b \quad \theta=(1,3,-4) / 8 \quad S O(9) \times S O(4) \\
& \Theta=[C(S O(9)), C(S O(4))] \\
& T_{2} \oplus T_{4} Z_{2} \text { fixed plane in the } \theta^{2} \text { twisted sector. } \\
& A_{1} \approx A_{2} \approx A_{3} \approx 2 A_{4} \approx 2 A_{5} \approx 2 A_{6} \approx 0 \\
& \text { B.20 } \quad Z_{8}-I I-c \quad \theta=(1,3,-4) / 8 \quad S O(8)^{[2]} \times S O(4) \\
& \Theta=\left[C\left(S O(8)^{[2]}\right), C(S O(4))\right] \\
& T_{2} \oplus T_{4} Z_{2} \text { fixed plane in the } \theta^{2} \text { twisted sector. } \\
& A_{1} \approx A_{2} \approx A_{3} \approx A_{4}, 2 A_{1} \approx 2 A_{5} \approx 2 A_{6} \approx 0 \\
& \text { B.21 } \quad Z_{12}-I-a \quad \theta=(1,-5,4) / 12 \quad E_{6} \\
& \Theta=\left[C\left(E_{6}\right)\right] \\
& \text { non- } T_{2} \oplus T_{4} Z_{3} \text { fixed plane in the } \theta^{3} \text { twisted sector. } \\
& A_{1} \approx A_{2} \approx A_{3} \approx A_{4} \approx A_{5}, 3 A_{5} \approx A_{6} \approx 0
\end{aligned}
$$$$
\text { B.22 } Z_{12}-I-b \quad \theta=(1,-5,4) / 12 \quad F_{4} \times S U(3)
$$$$
\Theta=\left[C\left(F_{4}\right), C(S U(3))\right]
$$$$
T_{2} \oplus T_{4} Z_{3} \text { fixed plane in the } \theta^{3} \text { twisted sector. }
$$$$
A_{1} \approx A_{2} \approx A_{3} \approx A_{4} \approx 0, A_{5} \approx A_{6}, 3 A_{6} \approx 0
$$

$$
\text { B.23 } Z_{12}-I-c \quad \theta=(1,-5,4) / 12 \quad S O(8)^{[3]} \times S U(3)
$$$$
\Theta=\left[C\left(S O(8)^{[3]}\right), C(S U(3))\right]
$$$$
T_{2} \oplus T_{4} Z_{3} \text { fixed plane in the } \theta^{3} \text { twisted sector. }
$$$$
A_{1} \approx A_{2} \approx A_{3} \approx A_{4} \approx 0, A_{5} \approx A_{6}, 3 A_{6} \approx 0
$$

$$
\text { B.24 } Z_{12}-I I-a \quad \theta=(1,5,-6) / 12 \quad F_{4} \times S O(4)
$$

$\Theta=\left[C\left(F_{4}\right), C(S O(4))\right]$

$T_{2} \oplus T_{4} Z_{2}$ fixed plane in the $\theta^{2}$ twisted sector.

$A_{1} \approx A_{2} \approx A_{3} \approx A_{4} \approx 0,2 A_{5} \approx 2 A_{6}$

$$
\begin{aligned}
& \text { B.25 } Z_{12}-I I-b \quad \theta=(1,5,-6) / 12 \quad S O(8)^{[3]} \times S O(4) \\
& \Theta=\left[C\left(S O(8)^{[3]}\right), C(S O(4))\right] \\
& T_{2} \oplus T_{4} Z_{2} \text { fixed plane in the } \theta^{2} \text { twisted sector. } \\
& A_{1} \approx A_{2} \approx A_{3} \approx A_{4} \approx 0,2 A_{5} \approx 2 A_{6}
\end{aligned}
$$


B.26 $Z_{2} \times Z_{2} \quad \theta=(1,0,-1) / 2 \quad \omega=(0,1,-1) / 2 \quad(S O(4))^{3}$ $\Theta=[C(S O(4)), I, C(S O(4))] \Omega=[I, C(S O(4)), C(S O(4))]$

$T_{2} \oplus T_{4}\left(I, Z_{2}\right),\left(Z_{2}, I\right)$ and $\left(Z_{2}, Z_{2}\right)$ fixed planes in the $\theta, \omega$ and $\theta \omega$ twisted sectors respectively.

$2 A_{1} \approx 2 A_{2} \approx 2 A_{3} \approx 2 A_{4} \approx 2 A_{5} \approx 2 A_{6} \approx 0$

B.27 $Z_{3} \times Z_{3} \quad \theta=(1,0,-1) / 3 \quad \omega=(0,1,-1) / 3 \quad(S U(3))^{3}$

$\Theta=[C(S U(3)), I, C(S U(3))] \Omega=[I, C(S U(3)), C(S U(3))]$

$T_{2} \oplus T_{4}\left(I, Z_{3}\right),\left(Z_{3}, I\right)$ and $\left(Z_{3}, Z_{3}\right)$ fixed planes in the $\theta^{k}, \omega^{k}$ and $\left(\theta \omega^{2}\right)^{k}$ twisted sectors respectively.

$A_{1} \approx A_{2}, A_{3} \approx A_{4}, A_{5} \approx A_{6}, 3 A_{1} \approx 3 A_{3} \approx 3 A_{5} \approx 0$

B.28 $Z_{4} \times Z_{4} \quad \theta=(1,0,-1) / 4 \quad \omega=(0,1,-1) / 4 \quad(S O(5))^{3}$

$\Theta=[C(S O(5)), I, C(S O(5))] \Omega=[I, C(S O(5)), C(S O(5))]$

$T_{2} \oplus T_{4}\left(I, Z_{4}\right),\left(Z_{4}, I\right)$ and $\left(Z_{4}, Z_{4}\right)$ fixed planes in the $\theta^{k}, \omega^{k}$ and $\left(\theta^{2} \omega^{2}\right)^{k}$ twisted sectors respectively.

$A_{1} \approx A_{3} \approx A_{5} \approx 0,2 A_{2} \approx 2 A_{4} \approx 2 A_{6} \approx 0$

B.29 $Z_{3} \times Z_{6}-a \quad \theta=(1,0,-1) / 3 \quad \omega=(0,1,-1) / 6 \quad(S U(3))^{3}$

$\Theta=[C(S U(3)), I, C(S U(3))] \Omega=\left[I, C\left(S U(3)^{[2]}\right), C\left(S U(3)^{[2]}\right)\right]$

$T_{2} \oplus T_{4}\left(I, Z_{6}\right),\left(Z_{3}, I\right)$ and $\left(Z_{3}, Z_{6}\right)$ fixed planes in the $\theta^{k}, \omega^{k}$ and $\left(\theta \omega^{4}\right)^{k}$ twisted sectors respectively.

$A_{1} \approx A_{2}, 3 A_{1} \approx 0, A_{3} \approx A_{4} \approx A_{5} \approx A_{6} \approx 0$

B.30 $Z_{3} \times Z_{6}-b \quad \theta=(1,0,-1) / 3 \quad \omega=(0,1,-1) / 6 \quad S U(3) \times$ $G_{2} \times S U(3)$

$\Theta=[C(S U(3)), I, C(S U(3))] \Omega=\left[I, C\left(G_{2}\right), C\left(S U(3)^{[2]}\right)\right]$

$T_{2} \oplus T_{4}\left(I, Z_{6}\right),\left(Z_{3}, I\right)$ and $\left(Z_{3}, Z_{6}\right)$ fixed planes in the $\theta^{k}, \omega^{k}$ and $\left(\theta \omega^{4}\right)^{k}$ twisted sectors respectively.

$A_{1} \approx A_{2}, 3 A_{1} \approx 0, A_{3} \approx A_{4} \approx A_{5} \approx A_{6} \approx 0$

B.31 $Z_{6} \times Z_{6}-a \quad \theta=(1,0,-1) / 6 \quad \omega=(0,1,-1) / 6 \quad\left(G_{2}\right)^{3}$

$\Theta=\left[C\left(G_{2}\right), I, C\left(G_{2}\right)\right] \Omega=\left[I, C\left(G_{2}\right), C\left(G_{2}\right)\right]$

$T_{2} \oplus T_{4}\left(I, Z_{6}\right),\left(Z_{6}, I\right)$ and $\left(Z_{6}, Z_{6}\right)$ fixed planes in the $\theta^{k}, \omega^{k}$ and $\left(\theta \omega^{5}\right)^{k}$ twisted sectors respectively. 
$A_{1} \approx A_{2} \approx A_{3} \approx A_{4} \approx A_{5} \approx A_{6} \approx 0$

B.32 $Z_{6} \times Z_{6}-b \quad \theta=(1,0,-1) / 6 \quad \omega=(0,1,-1) / 6 \quad\left(G_{2}\right)^{2} \times$ $S U(3)$

$\Theta=\left[C\left(G_{2}\right), I, C\left(S U(3)^{[2]}\right)\right] \quad \Omega=\left[I, C\left(G_{2}\right), C\left(S U(3)^{[2]}\right)\right]$

$T_{2} \oplus T_{4}\left(I, Z_{6}\right),\left(Z_{6}, I\right)$ and $\left(Z_{6}, Z_{6}\right)$ fixed planes in the $\theta^{k}, \omega^{k}$ and $\left(\theta \omega^{5}\right)^{k}$ twisted sectors respectively.

$A_{1} \approx A_{2} \approx A_{3} \approx A_{4} \approx A_{5} \approx A_{6} \approx 0$

B.33 $Z_{6} \times Z_{6}-c \quad \theta=(1,0,-1) / 6 \quad \omega=(0,1,-1) / 6 \quad G_{2} \times$ $(S U(3))^{2}$

$\Theta=\left[C\left(G_{2}\right), I, C\left(S U(3)^{[2]}\right)\right] \quad \Omega=\left[I, C\left(S U(3)^{[2]}\right), C\left(S U(3)^{[2]}\right)\right]$

$T_{2} \oplus T_{4}\left(I, Z_{6}\right),\left(Z_{6}, I\right)$ and $\left(Z_{6}, Z_{6}\right)$ fixed planes in the $\theta^{k}, \omega^{k}$ and $\left(\theta \omega^{5}\right)^{k}$ twisted sectors respectively.

$A_{1} \approx A_{2} \approx A_{3} \approx A_{4} \approx A_{5} \approx A_{6} \approx 0$

B.34 $Z_{6} \times Z_{6}-d \quad \theta=(1,0,-1) / 6 \quad \omega=(0,1,-1) / 6 \quad(S U(3))^{3}$

$\Theta=\left[C\left(S U(3)^{[2]}\right), I, C\left(S U(3)^{[2]}\right)\right] \quad \Omega=\left[I, C\left(S U(3)^{[2]}\right), C\left(S U(3)^{[2]}\right)\right]$

$T_{2} \oplus T_{4}\left(I, Z_{6}\right),\left(Z_{6}, I\right)$ and $\left(Z_{6}, Z_{6}\right)$ fixed planes in the $\theta^{k}, \omega^{k}$ and $\left(\theta \omega^{5}\right)^{k}$ twisted sectors respectively.

$A_{1} \approx A_{2} \approx A_{3} \approx A_{4} \approx A_{5} \approx A_{6} \approx 0$

\section{Modular Symmetries of New Orbifolds}

We now present the calculation of the modular symmetries and threshold corrections for $Z_{6}-I I-f$ without Wilson lines. The derivation uses the methods applied earlier [24, 25, 26] to Coxeter $Z_{N}$ orbifolds. The matrix $Q$ representing the action of the point group is $\Theta^{T}$ where

$$
\Theta=\left(C\left(S U(4)^{[2]}\right), C(S U(3)), C(S U(2))\right)
$$

as in appendix B. There is a non- $T_{2} \oplus T_{4} Z_{2}$ fixed plane in the $\theta^{2}$ twisted sector. Solving

$$
Q^{2} w=w
$$

and

$$
\left(Q^{*}\right)^{2} p=p
$$


where

$$
Q^{*} \equiv\left(Q^{T}\right)^{-1}
$$

gives the solutions $W_{\text {soln }}$ and $p_{\text {soln }}$ for the winding numbers and momenta in the $\theta^{2}$ twisted sector

$$
w_{\text {soln }}=\left(\begin{array}{c}
n_{1} \\
2 n_{1} \\
-n_{1} \\
0 \\
0 \\
n_{6}
\end{array}\right)
$$

and

$$
p_{\text {soln }}=\left(\begin{array}{c}
0 \\
m_{2} \\
-m_{2} \\
0 \\
0 \\
m_{6}
\end{array}\right)
$$

by The world sheet momentum $P$ is given by

$$
P=p_{\text {soln }}^{T} w_{\text {soln }}=m_{\perp}^{T} K^{T} n_{\perp}
$$

where

$$
m_{\perp}=\left(\begin{array}{l}
m_{2} \\
m_{6}
\end{array}\right), n_{\perp}=\left(\begin{array}{l}
n_{1} \\
n_{6}
\end{array}\right)
$$

Thus,

$$
K=\left(\begin{array}{ll}
3 & 0 \\
0 & 1
\end{array}\right)
$$

This matrix $K$ has the same form as for the $\theta^{2}$ twisted sector of the $Z_{6^{-}}$ II-c orbifold [25, 26] despite the very different form of the $w_{\text {soln }}$ and $p_{\text {soln }}$. Thus the modular symmetry group associated with the $Z_{2}$ fixed planes is $\Gamma^{0}(3)_{T} \times \Gamma_{0}(3)_{U}$ in the notation of earlier work [24, 25, 26] for congruence subgroups of $\operatorname{PSL}(2, \mathrm{Z})$, where the first factor refers to the $T$ modulus and the second factor to the $U$ modulus.

In addition, because we are dealing with the same orbifold appart from the lattice, the fundamental sectors and the construction of the renormalisation 
group co-efficient $b_{a}^{N=2}$ from the co-efficients $b_{a}^{\left(I, \theta^{2}\right)}, b_{a}^{\left(\theta^{4}, \theta^{2}\right)}, b_{a}^{\left(\theta^{4}, \theta^{4}\right)}$ and $b_{a}^{\left(\theta^{2}, I\right)}$ for the various twisted sectors is the same as for $Z_{6}-\mathrm{II}-\mathrm{c}$. With $K$ as above, the matrix $A$ involved in the construction of the partition function is

$$
A=\left(\begin{array}{cc}
n_{1} & l_{1} / 3 \\
n_{2} & l_{2}
\end{array}\right)
$$

just as for $Z_{6}-\mathrm{II}-\mathrm{c}$, and we obtain for the contribution of these sectors to the threshold correction

$\Delta_{a}=-2 b_{a}^{\left(I, \theta^{2}\right)} \ln \left(k T_{2}|\eta(T)|^{4} U_{2}|\eta(U)|^{4}\right)-2 b_{a}^{\left(I, \theta^{2}\right)} \ln \left(k T_{2}|\eta(T / 3)|^{4} U_{2}|\eta(3 U)|^{4}\right)(54)$

\section{Modular Subgroups for $T_{2} \oplus T_{4}$ Orbifolds}

We now list all the obtainable modular symmetries for $Z_{2}, Z, 3$ and $Z_{4}$ planes, where the plane lies completely in the $T^{2}$ sublattice, for all choices of Wilson lines consistent with modular and point group invariance. For each modular group we also provide an example Wilson line which yields this symmetry.

\section{D.1 $Z_{2}$ Plane}

For the $T$ modulus there are 7 possible modular groups:

\section{1. $\operatorname{PSL}(2, \mathrm{Z})$}

$$
A=\mathbf{0}
$$

2. $a, d=1 \bmod 4, c=0 \bmod 4$ and $a, d=3 \bmod 4, c=0 \bmod 4$

$$
A=\frac{1}{2}\left(\begin{array}{cccccccccccccccc}
0 & 0 & 0 & 0 & 0 & 0 & 0 & 0 & 0 & 0 & 0 & 0 & 0 & 0 & 0 & 0 \\
0 & 0 & 0 & 0 & 0 & 0 & 0 & 0 & 1 & 1 & 0 & 0 & 0 & 0 & 0 & 0
\end{array}\right)
$$

3. $a, d=1 \bmod 2, c=0 \bmod 2$

$$
A=\frac{1}{2}\left(\begin{array}{cccccccccccccccc}
0 & 0 & 0 & 0 & 0 & 0 & 0 & 0 & 0 & 0 & 0 & 0 & 0 & 0 & 0 & 0 \\
0 & 0 & 0 & 0 & 0 & 0 & 0 & 0 & 2 & 0 & 0 & 0 & 0 & 0 & 0 & 0
\end{array}\right)
$$

4. $a, d=1 \bmod 4, c=0 \bmod 16$ and $a, d=3 \bmod 4, c=0 \bmod 16$

$$
A=\frac{1}{2}\left(\begin{array}{llllllllllllllll}
1 & 1 & 0 & 0 & 0 & 0 & 0 & 0 & 0 & 0 & 0 & 0 & 0 & 0 & 0 & 0 \\
0 & 0 & 0 & 0 & 0 & 0 & 0 & 0 & 1 & 1 & 0 & 0 & 0 & 0 & 0 & 0
\end{array}\right)
$$

5. $a, d=1 \bmod 4, c=0 \bmod 8$ and $a, d=3 \bmod 4, c=0 \bmod 8$

$$
A=\frac{1}{2}\left(\begin{array}{cccccccccccccccc}
1 & 1 & 0 & 0 & 0 & 0 & 0 & 0 & 0 & 0 & 0 & 0 & 0 & 0 & 0 & 0 \\
0 & 0 & 0 & 0 & 0 & 0 & 0 & 0 & 2 & 0 & 0 & 0 & 0 & 0 & 0 & 0
\end{array}\right)
$$

6. $a, d=1 \bmod 2, c=0 \bmod 4$

$$
A=\frac{1}{2}\left(\begin{array}{cccccccccccccccc}
1 & 1 & 0 & 0 & 0 & 0 & 0 & 0 & 1 & 1 & 0 & 0 & 0 & 0 & 0 & 0 \\
0 & 0 & 0 & 0 & 0 & 0 & 0 & 0 & 0 & 0 & 2 & 0 & 0 & 0 & 0 & 0
\end{array}\right)
$$

7. $a, d=1 \bmod 4, c=0 \bmod 16$ and $a=1 \bmod 4, d=3 \bmod 4, c=$ $8 \bmod 16$ and $a, d=3 \bmod 4, c=0 \bmod 16$ and $a=3 \bmod 4, d=1 \bmod 4$, $c=8 \bmod 16$ 


$$
A=\frac{1}{2}\left(\begin{array}{llllllllllllllll}
1 & 1 & 0 & 0 & 0 & 0 & 0 & 0 & 1 & 1 & 0 & 0 & 0 & 0 & 0 & 0 \\
1 & 0 & 1 & 0 & 0 & 0 & 0 & 0 & 1 & 0 & 1 & 0 & 0 & 0 & 0 & 0
\end{array}\right)
$$

For the $U$ modulus there are 10 modular groups:

\section{1. $\operatorname{PSL}(2, Z)$}

$$
A=\mathbf{0}
$$

2. $a, d=1 \bmod 4, c=0 \bmod 4$ and $a, d=3 \bmod 4, c=0 \bmod 4$

$$
A=\frac{1}{2}\left(\begin{array}{cccccccccccccccc}
0 & 0 & 0 & 0 & 0 & 0 & 0 & 0 & 0 & 0 & 0 & 0 & 0 & 0 & 0 & 0 \\
0 & 0 & 0 & 0 & 0 & 0 & 0 & 0 & 1 & 1 & 0 & 0 & 0 & 0 & 0 & 0
\end{array}\right)
$$

3. $a, d=1 \bmod 2, c=0 \bmod 2$

$$
A=\frac{1}{2}\left(\begin{array}{cccccccccccccccc}
0 & 0 & 0 & 0 & 0 & 0 & 0 & 0 & 0 & 0 & 0 & 0 & 0 & 0 & 0 & 0 \\
0 & 0 & 0 & 0 & 0 & 0 & 0 & 0 & 2 & 0 & 0 & 0 & 0 & 0 & 0 & 0
\end{array}\right)
$$

4. $a, d=1 \bmod 4, b=0 \bmod 4$ and $a, d=3 \bmod 4, b=0 \bmod 4$

$$
A=\frac{1}{2}\left(\begin{array}{cccccccccccccccc}
1 & 1 & 0 & 0 & 0 & 0 & 0 & 0 & 0 & 0 & 0 & 0 & 0 & 0 & 0 & 0 \\
0 & 0 & 0 & 0 & 0 & 0 & 0 & 0 & 0 & 0 & 0 & 0 & 0 & 0 & 0 & 0
\end{array}\right)
$$

5. $a, d=1 \bmod 4, b, c=0 \bmod 4$ and $a, d=1 \bmod 4, b, c=2 \bmod 4$ and $a, d=3 \bmod 4, b, c=0 \bmod 4$ and $a, d=3 \bmod 4, b, c=2 \bmod 4$

$$
A=\frac{1}{2}\left(\begin{array}{cccccccccccccccc}
1 & 1 & 0 & 0 & 0 & 0 & 0 & 0 & 0 & 0 & 0 & 0 & 0 & 0 & 0 & 0 \\
0 & 0 & 0 & 0 & 0 & 0 & 0 & 0 & 1 & 1 & 0 & 0 & 0 & 0 & 0 & 0
\end{array}\right)
$$

6. $a, d=1 \bmod 4, b=0 \bmod 4, c=0 \bmod 2$ and $a, d=3 \bmod 4, b=$ $0 \bmod 4, c=0 \bmod 2$

$$
A=\frac{1}{2}\left(\begin{array}{cccccccccccccccc}
1 & 1 & 0 & 0 & 0 & 0 & 0 & 0 & 0 & 0 & 0 & 0 & 0 & 0 & 0 & 0 \\
0 & 0 & 0 & 0 & 0 & 0 & 0 & 0 & 2 & 0 & 0 & 0 & 0 & 0 & 0 & 0
\end{array}\right)
$$

7. $a, d=1 \bmod 2, b=0 \bmod 2$

$$
A=\frac{1}{2}\left(\begin{array}{llllllllllllllll}
1 & 1 & 0 & 0 & 0 & 0 & 0 & 0 & 1 & 1 & 0 & 0 & 0 & 0 & 0 & 0 \\
0 & 0 & 0 & 0 & 0 & 0 & 0 & 0 & 0 & 0 & 0 & 0 & 0 & 0 & 0 & 0
\end{array}\right)
$$

8. $a, d=1 \bmod 4, b=0 \bmod 2, c=0 \bmod 4$ and $a, d=3 \bmod 4, b=$ $0 \bmod 2, c=0 \bmod 4$

$$
A=\frac{1}{4}\left(\begin{array}{cccccccccccccccc}
2 & 2 & 0 & 0 & 0 & 0 & 0 & 0 & 2 & 2 & 0 & 0 & 0 & 0 & 0 & 0 \\
0 & 0 & 0 & 0 & 0 & 0 & 0 & 0 & 1 & -1 & -1 & -1 & -1 & 1 & 1 & 1
\end{array}\right)
$$

9. $a, d=1 \bmod 2, b, c=0 \bmod 2$

$$
A=\frac{1}{2}\left(\begin{array}{cccccccccccccccc}
1 & 1 & 0 & 0 & 0 & 0 & 0 & 0 & 1 & 1 & 0 & 0 & 0 & 0 & 0 & 0 \\
0 & 0 & 0 & 0 & 0 & 0 & 0 & 0 & 2 & 0 & 0 & 0 & 0 & 0 & 0 & 0
\end{array}\right)
$$

10. $a, d=1 \bmod 2, b, c=0 \bmod 2$ and $a, d=0 \bmod 2, b, c=1 \bmod 2$

$$
A=\frac{1}{2}\left(\begin{array}{cccccccccccccccc}
2 & 0 & 0 & 0 & 0 & 0 & 0 & 0 & 0 & 0 & 0 & 0 & 0 & 0 & 0 & 0 \\
2 & 0 & 0 & 0 & 0 & 0 & 0 & 0 & 0 & 0 & 0 & 0 & 0 & 0 & 0 & 0
\end{array}\right)
$$

\section{D.2 $Z_{3}$ Plane}

The $U$ modulus is fixed for a $Z_{3}$ plane. For the $T$ modulus there are 2 modular groups:

1. $\operatorname{PSL}(2, \mathrm{Z})$

$$
A=\mathbf{0}
$$

2. $a, d=1 \bmod 3, c=0 \bmod 3$ 


$$
A=\frac{1}{3}\left(\begin{array}{cccccccccccccccc}
2 & 1 & 1 & 0 & 0 & 0 & 0 & 0 & 0 & 0 & 0 & 0 & 0 & 0 & 0 & 0 \\
2 & 1 & 1 & 0 & 0 & 0 & 0 & 0 & 0 & 0 & 0 & 0 & 0 & 0 & 0 & 0
\end{array}\right)
$$

\section{D.3 $Z_{4}$ Plane}

The $U$ modulus is fixed for a $Z_{4}$ plane. For the $T$ modulus there are 2 modular groups:

1. $\operatorname{PSL}(2, Z)$

$$
A=\mathbf{0}
$$

2. $\Gamma_{0}(2)$

$$
A=\frac{1}{2}\left(\begin{array}{cccccccccccccccc}
0 & 0 & 0 & 0 & 0 & 0 & 0 & 0 & 0 & 0 & 0 & 0 & 0 & 0 & 0 & 0 \\
2 & 0 & 0 & 0 & 0 & 0 & 0 & 0 & 0 & 0 & 0 & 0 & 0 & 0 & 0 & 0
\end{array}\right)
$$

\section{E Modular Subgroups for non- $T_{2} \oplus T_{4}$ Orb- ifolds}

We now list all the obtainable modular symmetries of fixed planes, where the orbifold cannot be decomposed as $T_{2} \oplus T_{4}$ for all choices of Wilson lines consistent with modular and point group invariance. For each modular group we also provide an example Wilson line which yields this symmetry.

\section{E.1 $Z_{4}-\mathbf{a}\left(S U(4)^{2}\right)$}

There is a non- $T_{2} \oplus T_{4} Z_{2}$ fixed plane in the $\theta^{2}$ twisted sector.

$R=\left(\begin{array}{ll}2 & 0 \\ 0 & 2\end{array}\right) \quad K=\left(\begin{array}{ll}2 & 0 \\ 0 & 2\end{array}\right)$

Wilson lines as stated here correspond to thes six component line as: $A=$ $\left(\begin{array}{l}A_{1} \\ A_{4}\end{array}\right)$

For the $T$ modulus there are 6 modular groups:

1. $\Gamma^{0}(2)$

$$
A=\mathbf{0}
$$

2. $a=1 \bmod 4, b, c=0 \bmod 2, d=1 \bmod 2$

$$
A=\frac{1}{4}\left(\begin{array}{cccccccccccccccc}
0 & 0 & 0 & 0 & 0 & 0 & 0 & 0 & 0 & 0 & 0 & 0 & 0 & 0 & 0 & 0 \\
2 & 1 & 1 & 1 & 1 & 0 & 0 & 0 & 0 & 0 & 0 & 0 & 0 & 0 & 0 & 0
\end{array}\right)
$$

3. $a=1 \bmod 4, b=0 \bmod 2, c=0 \bmod 4, d=1 \bmod 2$ and $a=3 \bmod 4$, $b=0 \bmod 2, c=2 \bmod 4, d=1 \bmod 2$

$$
A=\frac{1}{4}\left(\begin{array}{cccccccccccccccc}
2 & 0 & 0 & 0 & 0 & 0 & 0 & 0 & 2 & 0 & 0 & 0 & 0 & 0 & 0 & 0 \\
0 & 2 & 2 & 2 & 0 & 0 & 0 & 0 & 0 & 2 & 2 & 2 & 0 & 0 & 0 & 0
\end{array}\right)
$$

4. $a=1 \bmod 4, b=0 \bmod 2, c=0 \bmod 4, d=1 \bmod 2$

$$
A=\frac{1}{4}\left(\begin{array}{cccccccccccccccc}
2 & 1 & 1 & 0 & 0 & 0 & 0 & 0 & 1 & 1 & 0 & 0 & 0 & 0 & 0 & 0 \\
0 & 0 & 0 & 0 & 0 & 0 & 0 & 0 & 1 & -1 & 1 & 1 & 1 & 1 & 1 & 1
\end{array}\right)
$$


5. $a, d=1 \bmod 2, b, c=0 \bmod 2$

$$
A=\frac{1}{4}\left(\begin{array}{cccccccccccccccc}
2 & 2 & 0 & 0 & 0 & 0 & 0 & 0 & 0 & 0 & 0 & 0 & 0 & 0 & 0 & 0 \\
0 & 0 & 0 & 0 & 0 & 0 & 0 & 0 & 2 & 2 & 0 & 0 & 0 & 0 & 0 & 0
\end{array}\right)
$$

6. $a, d=0 \bmod 2, b, c=1 \bmod 2$ and $a, d=1 \bmod 2, b, c=0 \bmod 2$

$$
A=\frac{1}{4}\left(\begin{array}{cccccccccccccccc}
2 & 2 & 0 & 0 & 0 & 0 & 0 & 0 & 2 & 2 & 0 & 0 & 0 & 0 & 0 & 0 \\
-1 & -1 & 3 & -1 & -1 & 1 & 1 & -1 & -1 & -1 & 3 & -1 & -1 & 1 & 1 & -1
\end{array}\right)
$$

For the $U$ modulus there are 9 modular groups:

$$
\text { 1. } \operatorname{PSL}(2, \mathrm{Z})
$$

$$
A=\mathbf{0}
$$

2. $a, d=1 \bmod 2, c=0 \bmod 2$

$$
A=\frac{1}{4}\left(\begin{array}{cccccccccccccccc}
0 & 0 & 0 & 0 & 0 & 0 & 0 & 0 & 0 & 0 & 0 & 0 & 0 & 0 & 0 & 0 \\
2 & 2 & 0 & 0 & 0 & 0 & 0 & 0 & 0 & 0 & 0 & 0 & 0 & 0 & 0 & 0
\end{array}\right)
$$

3. $a=1 \bmod 4, c=0 \bmod 2, d=1 \bmod 2$

$$
A=\frac{1}{4}\left(\begin{array}{cccccccccccccccc}
0 & 0 & 0 & 0 & 0 & 0 & 0 & 0 & 0 & 0 & 0 & 0 & 0 & 0 & 0 & 0 \\
2 & 1 & 1 & 1 & 1 & 0 & 0 & 0 & 0 & 0 & 0 & 0 & 0 & 0 & 0 & 0
\end{array}\right)
$$

4. $a=1 \bmod 2, b=0 \bmod 4, d=1 \bmod 4$

$$
A=\frac{1}{4}\left(\begin{array}{cccccccccccccccc}
2 & 0 & 0 & 0 & 0 & 0 & 0 & 0 & 2 & 0 & 0 & 0 & 0 & 0 & 0 & 0 \\
0 & 0 & 0 & 0 & 0 & 0 & 0 & 0 & 0 & 0 & 0 & 0 & 0 & 0 & 0 & 0
\end{array}\right)
$$

5. $a, d=1 \bmod 4, b, c=0 \bmod 2$ and $a=3 \bmod 4, b=2 \bmod 4, c=$ $0 \bmod 4, d=1 \bmod 4$ and $a=1 \bmod 4, b=0 \bmod 4, c=2 \bmod 4, d=$ $3 \bmod 4$ and $a=3 \bmod 4, b=2 \bmod 4, c=2 \bmod 4, d=3 \bmod 4$

$$
A=\frac{1}{4}\left(\begin{array}{cccccccccccccccc}
2 & 0 & 0 & 0 & 0 & 0 & 0 & 0 & 2 & 0 & 0 & 0 & 0 & 0 & 0 & 0 \\
0 & 2 & 2 & 2 & 0 & 0 & 0 & 0 & 0 & 2 & 2 & 2 & 0 & 0 & 0 & 0
\end{array}\right)
$$

6. $a=1 \bmod 2, b=0 \bmod 4, c=0 \bmod 2, d=1 \bmod 4$

$$
A=\frac{1}{4}\left(\begin{array}{ccccccccccccccccc}
2 & 1 & 1 & 0 & 0 & 0 & 0 & 0 & 1 & 1 & 0 & 0 & 0 & 0 & 0 & 0 \\
0 & 0 & 0 & 0 & 0 & 0 & 0 & 0 & 1 & -1 & 1 & 1 & 1 & 1 & 1 & 1
\end{array}\right)
$$

7. $a, d=1 \bmod 4, b, d=0 \bmod 4$

$$
A=\frac{1}{8}\left(\begin{array}{cccccccccccccccc}
4 & 2 & 2 & 0 & 0 & 0 & 0 & 0 & 2 & 2 & 0 & 0 & 0 & 0 & 0 & 0 \\
-1 & 1 & 1 & -1 & -1 & 1 & 1 & -1 & 0 & 0 & 2 & -2 & -2 & 2 & 2 & -2
\end{array}\right)
$$

8. $a, d=1 \bmod 2, b=0 \bmod 2$

$$
A=\frac{1}{4}\left(\begin{array}{cccccccccccccccc}
2 & 2 & 0 & 0 & 0 & 0 & 0 & 0 & 0 & 0 & 0 & 0 & 0 & 0 & 0 & 0 \\
0 & 0 & 0 & 0 & 0 & 0 & 0 & 0 & 0 & 0 & 0 & 0 & 0 & 0 & 0 & 0
\end{array}\right)
$$

9. $a, d=1 \bmod 2, b, c=0 \bmod 2$

$$
A=\frac{1}{4}\left(\begin{array}{llllllllllllllll}
2 & 2 & 0 & 0 & 0 & 0 & 0 & 0 & 0 & 0 & 0 & 0 & 0 & 0 & 0 & 0 \\
0 & 0 & 0 & 0 & 0 & 0 & 0 & 0 & 2 & 2 & 0 & 0 & 0 & 0 & 0 & 0
\end{array}\right)
$$

\section{E.2 $Z_{4}-\mathbf{b} S U(4) \times S O(5) \times S U(2)$}

There is a non- $T_{2} \oplus T_{4} Z_{2}$ fixed plane in the $\theta^{2}$ twisted sector.

$R=\left(\begin{array}{ll}2 & 0 \\ 0 & 1\end{array}\right) \quad K=\left(\begin{array}{ll}2 & 0 \\ 0 & 1\end{array}\right)$

Wilson lines as stated here correspond to thes six component line as: $A=$ $\left(\begin{array}{l}A_{1} \\ A_{6}\end{array}\right)$ 
For the $T$ modulus there are 9 modular groups:

\section{1. $\Gamma^{0}(2)$}

$$
A=\mathbf{0}
$$

2. $a, d=1 \bmod 4, b=0 \bmod 2, c=0 \bmod 4$ and $a, d=3 \bmod 4, b=$ $0 \bmod 2, c=0 \bmod 4$

$$
A=\frac{1}{2}\left(\begin{array}{cccccccccccccccc}
0 & 0 & 0 & 0 & 0 & 0 & 0 & 0 & 0 & 0 & 0 & 0 & 0 & 0 & 0 & 0 \\
1 & 1 & 0 & 0 & 0 & 0 & 0 & 0 & 0 & 0 & 0 & 0 & 0 & 0 & 0 & 0
\end{array}\right)
$$

3. $a=1 \bmod 2, b, c=0 \bmod 2$

$$
A=\frac{1}{4}\left(\begin{array}{cccccccccccccccc}
0 & 0 & 0 & 0 & 0 & 0 & 0 & 0 & 0 & 0 & 0 & 0 & 0 & 0 & 0 & 0 \\
2 & 0 & 0 & 0 & 0 & 0 & 0 & 0 & 0 & 0 & 0 & 0 & 0 & 0 & 0 & 0
\end{array}\right)
$$

4. $a, d=1 \bmod 4, b, c=0 \bmod 2$

$$
A=\frac{1}{4}\left(\begin{array}{cccccccccccccccc}
2 & 0 & 0 & 0 & 0 & 0 & 0 & 0 & 2 & 0 & 0 & 0 & 0 & 0 & 0 & 0 \\
0 & 0 & 0 & 0 & 0 & 0 & 0 & 0 & 0 & 0 & 0 & 0 & 0 & 0 & 0 & 0
\end{array}\right)
$$

5. $a, d=1 \bmod 4, b=0 \bmod 2, c=0 \bmod 8$

$$
A=\frac{1}{4}\left(\begin{array}{llllllllllllllll}
2 & 0 & 0 & 0 & 0 & 0 & 0 & 0 & 2 & 0 & 0 & 0 & 0 & 0 & 0 & 0 \\
0 & 1 & 1 & 0 & 0 & 0 & 0 & 0 & 0 & 0 & 0 & 0 & 0 & 0 & 0 & 0
\end{array}\right)
$$

6. $a=1 \bmod 4, b=0 \bmod 2, c=0 \bmod 4, d=1 \bmod 2$

$$
A=\frac{1}{4}\left(\begin{array}{llllllllllllllll}
2 & 0 & 0 & 0 & 0 & 0 & 0 & 0 & 2 & 0 & 0 & 0 & 0 & 0 & 0 & 0 \\
0 & 2 & 0 & 0 & 0 & 0 & 0 & 0 & 0 & 0 & 0 & 0 & 0 & 0 & 0 & 0
\end{array}\right)
$$

7. $a=1 \bmod 4, b=0 \bmod 2, c=0 \bmod 4, d=1 \bmod 2$ and $a=3 \bmod 4$, $b=0 \bmod 2, c=2 \bmod 4, d=1 \bmod 2$

$$
A=\frac{1}{4}\left(\begin{array}{cccccccccccccccc}
2 & 0 & 0 & 0 & 0 & 0 & 0 & 0 & 2 & 0 & 0 & 0 & 0 & 0 & 0 & 0 \\
0 & 2 & 0 & 0 & 0 & 0 & 0 & 0 & 0 & 2 & 0 & 0 & 0 & 0 & 0 & 0
\end{array}\right)
$$

8. $a, d=1 \bmod 4, b=0 \bmod 2, c=0 \bmod 8$ and $a, d=3 \bmod 4, b=$ $0 \bmod 2, c=0 \bmod 8$ and $a, d=1 \bmod 4, b=1 \bmod 2, c=4 \bmod 8$ and $a, d=3 \bmod 4, b=1 \bmod 2, c=4 \bmod 8$

$$
A=\frac{1}{4}\left(\begin{array}{cccccccccccccccc}
2 & 2 & 0 & 0 & 0 & 0 & 0 & 0 & 0 & 0 & 0 & 0 & 0 & 0 & 0 & 0 \\
0 & 0 & 0 & 0 & 0 & 0 & 0 & 0 & 1 & 1 & 0 & 0 & 0 & 0 & 0 & 0
\end{array}\right)
$$

9. $a, d=1 \bmod 2, b=0 \bmod 2, c=0 \bmod 4$

$$
A=\frac{1}{4}\left(\begin{array}{cccccccccccccccc}
2 & 2 & 0 & 0 & 0 & 0 & 0 & 0 & 0 & 0 & 0 & 0 & 0 & 0 & 0 & 0 \\
0 & 0 & 0 & 0 & 0 & 0 & 0 & 0 & 2 & 0 & 0 & 0 & 0 & 0 & 0 & 0
\end{array}\right)
$$

\section{E.3 $\quad Z_{6}-\mathbf{I I}-\mathbf{a} S U(6) \times S U(2)$}

There is a non- $T_{2} \oplus T_{4} Z_{2}$ fixed plane in the $\theta^{2}$ twisted sector and a non$T_{2} \oplus T_{4} Z_{3}$ fixed plane in the $\theta^{3}$ twisted sector.

$R=\left(\begin{array}{ll}3 & 0 \\ 0 & 1\end{array}\right) \quad K=\left(\begin{array}{ll}3 & 0 \\ 0 & 1\end{array}\right)$

Wilson lines as stated here correspond to thes six component line as: $A=$ $\left(\begin{array}{l}A_{1} \\ A_{6}\end{array}\right)$

The $\theta^{2}$ sector:

For the $T$ modulus there are 6 modular groups:

1. $\Gamma^{0}(3)$ 


$$
A=\mathbf{0}
$$

2. $a, d=1 \bmod 4, b=0 \bmod 3, c=0 \bmod 4$ and $a, d=3 \bmod 4, b=$ $0 \bmod 3, c=0 \bmod 4$

$$
A=\frac{1}{2}\left(\begin{array}{cccccccccccccccc}
0 & 0 & 0 & 0 & 0 & 0 & 0 & 0 & 0 & 0 & 0 & 0 & 0 & 0 & 0 & 0 \\
1 & 1 & 0 & 0 & 0 & 0 & 0 & 0 & 0 & 0 & 0 & 0 & 0 & 0 & 0 & 0
\end{array}\right)
$$

3. $a, d=1 \bmod 2, b=0 \bmod 3, c=0 \bmod 2$

$$
A=\frac{1}{2}\left(\begin{array}{cccccccccccccccc}
0 & 0 & 0 & 0 & 0 & 0 & 0 & 0 & 0 & 0 & 0 & 0 & 0 & 0 & 0 & 0 \\
2 & 0 & 0 & 0 & 0 & 0 & 0 & 0 & 0 & 0 & 0 & 0 & 0 & 0 & 0 & 0
\end{array}\right)
$$

4. $a=1 \bmod 6, b=0 \bmod 3, c=0 \bmod 2, d=1 \bmod 2$

$$
A=\frac{1}{6}\left(\begin{array}{cccccccccccccccc}
2 & 1 & 1 & 0 & 0 & 0 & 0 & 0 & 2 & 1 & 1 & 0 & 0 & 0 & 0 & 0 \\
0 & 0 & 0 & 0 & 0 & 0 & 0 & 0 & 0 & 0 & 0 & 0 & 0 & 0 & 0 & 0
\end{array}\right)
$$

5. $a=1 \bmod 12, b=0 \bmod 3, c=0 \bmod 8, d=1 \bmod 4$ and $a=7 \bmod 12$, $b=0 \bmod 3, c=0 \bmod 8, d=3 \bmod 4$

$$
A=\frac{1}{12}\left(\begin{array}{cccccccccccccccc}
4 & 2 & 2 & 0 & 0 & 0 & 0 & 0 & 4 & 2 & 2 & 0 & 0 & 0 & 0 & 0 \\
0 & 0 & 0 & 0 & 0 & 0 & 0 & 0 & -3 & 3 & 3 & -3 & -3 & 3 & 3 & -3
\end{array}\right)
$$

6. $a=1 \bmod 6, b=0 \bmod 3, c=0 \bmod 8, d=1 \bmod 2$

$$
A=\frac{1}{12}\left(\begin{array}{cccccccccccccccc}
4 & 2 & 2 & 0 & 0 & 0 & 0 & 0 & 4 & 2 & 2 & 0 & 0 & 0 & 0 & 0 \\
0 & 0 & 0 & 0 & 0 & 0 & 0 & 0 & -3 & 9 & -3 & -3 & -3 & 3 & 3 & -3
\end{array}\right)
$$

For the $U$ modulus there are 7 modular groups:

1. $\Gamma_{0}(3)$

$$
A=\mathbf{0}
$$

2. $a, d=1 \bmod 4, c=0 \bmod 12$ and $a, d=3 \bmod 4, c=0 \bmod 12$

$$
A=\frac{1}{2}\left(\begin{array}{llllllllllllllll}
0 & 0 & 0 & 0 & 0 & 0 & 0 & 0 & 0 & 0 & 0 & 0 & 0 & 0 & 0 & 0 \\
1 & 1 & 0 & 0 & 0 & 0 & 0 & 0 & 0 & 0 & 0 & 0 & 0 & 0 & 0 & 0
\end{array}\right)
$$

3. $a, d=1 \bmod 2, c=0 \bmod 6$

$$
A=\frac{1}{2}\left(\begin{array}{cccccccccccccccc}
0 & 0 & 0 & 0 & 0 & 0 & 0 & 0 & 0 & 0 & 0 & 0 & 0 & 0 & 0 & 0 \\
2 & 0 & 0 & 0 & 0 & 0 & 0 & 0 & 0 & 0 & 0 & 0 & 0 & 0 & 0 & 0
\end{array}\right)
$$

4. $a=1 \bmod 6, b=0 \bmod 2, c=0 \bmod 3, d=1 \bmod 12$

$$
A=\frac{1}{6}\left(\begin{array}{cccccccccccccccc}
2 & 1 & 1 & 0 & 0 & 0 & 0 & 0 & 2 & 1 & 1 & 0 & 0 & 0 & 0 & 0 \\
0 & 0 & 0 & 0 & 0 & 0 & 0 & 0 & 0 & 0 & 0 & 0 & 0 & 0 & 0 & 0
\end{array}\right)
$$

5. $a=1 \bmod 4, b=0 \bmod 2, c=0 \bmod 12, d=1 \bmod 12$ and $a=3 \bmod 4$, $b=0 \bmod 2, c=0 \bmod 12, d=7 \bmod 12$

$$
A=\frac{1}{12}\left(\begin{array}{cccccccccccccccc}
4 & 2 & 2 & 0 & 0 & 0 & 0 & 0 & 4 & 2 & 2 & 0 & 0 & 0 & 0 & 0 \\
0 & 0 & 0 & 0 & 0 & 0 & 0 & 0 & -3 & 3 & 3 & -3 & -3 & 3 & 3 & -3
\end{array}\right)
$$

6. $a=1 \bmod 2, b=0 \bmod 2, c=0 \bmod 6, d=1 \bmod 6$

$$
A=\frac{1}{12}\left(\begin{array}{cccccccccccccccc}
4 & 2 & 2 & 0 & 0 & 0 & 0 & 0 & 4 & 2 & 2 & 0 & 0 & 0 & 0 & 0 \\
0 & 0 & 0 & 0 & 0 & 0 & 0 & 0 & -3 & 9 & -3 & -3 & -3 & 3 & 3 & -3
\end{array}\right)
$$

7. $a=1 \bmod 2, b=0 \bmod 2, c=0 \bmod 6, d=1 \bmod 6$ and $a=0 \bmod 2$, $b=1 \bmod 2, c=3 \bmod 6, d=4 \bmod 6$

$$
A=\frac{1}{6}\left(\begin{array}{cccccccccccccccc}
2 & 2 & 2 & 0 & 0 & 0 & 0 & 0 & 0 & 0 & 0 & 0 & 0 & 0 & 0 & 0 \\
0 & 0 & 0 & 6 & 0 & 0 & 0 & 0 & 0 & 0 & 0 & 0 & 0 & 0 & 0 & 0
\end{array}\right)
$$

The $\theta^{3}$ sector:

$$
R=\left(\begin{array}{ll}
2 & 0 \\
0 & 2
\end{array}\right) \quad K=\left(\begin{array}{ll}
2 & 0 \\
0 & 2
\end{array}\right)
$$


Wilson lines as stated here correspond to thes six component line as: $A=$ $\left(\begin{array}{l}A_{1} \\ A_{4}\end{array}\right)$

For the $T$ modulus there are 3 modular groups:

1. $\Gamma^{0}(2)$

$$
A=\mathbf{0}
$$

2. $a=1 \bmod 6, b=0 \bmod 2, c=0 \bmod 3, d=1 \bmod 3$

$$
A=\frac{1}{6}\left(\begin{array}{llllllllllllllll}
2 & 1 & 1 & 0 & 0 & 0 & 0 & 0 & 2 & 1 & 1 & 0 & 0 & 0 & 0 & 0 \\
2 & 1 & 1 & 0 & 0 & 0 & 0 & 0 & 2 & 1 & 1 & 0 & 0 & 0 & 0 & 0
\end{array}\right)
$$

3. $a, d=1 \bmod 3, b=0 \bmod 2, c=0 \bmod 3$

$$
A=\frac{1}{6}\left(\begin{array}{llllllllllllllll}
4 & 0 & 0 & 0 & 0 & 0 & 0 & 0 & 2 & 2 & 0 & 0 & 0 & 0 & 0 & 0 \\
4 & 0 & 0 & 0 & 0 & 0 & 0 & 0 & 2 & 2 & 0 & 0 & 0 & 0 & 0 & 0
\end{array}\right)
$$

\section{E.4 $\quad Z_{6}-\mathbf{I I}-\mathbf{b} S U(3) \times S O(8)$}

There is a non- $T_{2} \oplus T_{4} Z_{2}$ fixed plane in the $\theta^{2}$ twisted sector.

$R=\left(\begin{array}{cc}0 & -1 \\ -1 & 0\end{array}\right) \quad K=\left(\begin{array}{ll}2 & 1 \\ 1 & 2\end{array}\right)$

Wilson lines as stated here correspond to thes six component line as: $A=$ $\left(\begin{array}{l}A_{5} \\ A_{6}\end{array}\right)$

For the $T$ modulus there are 4 modular groups:

1. $\Gamma^{0}(3)$

$$
A=\mathbf{0}
$$

2. $a, d=1 \bmod 2, b=0 \bmod 3, c=0 \bmod 2$

$$
A=\frac{1}{2}\left(\begin{array}{cccccccccccccccc}
0 & 0 & 0 & 0 & 0 & 0 & 0 & 0 & 0 & 0 & 0 & 0 & 0 & 0 & 0 & 0 \\
2 & 0 & 0 & 0 & 0 & 0 & 0 & 0 & 0 & 0 & 0 & 0 & 0 & 0 & 0 & 0
\end{array}\right)
$$

3. $a, d=1 \bmod 4, b=0 \bmod 3, c=0 \bmod 8$ and $a, d=3 \bmod 4, b=$ $0 \bmod 3, c=0 \bmod 8$

$$
A=\frac{1}{2}\left(\begin{array}{cccccccccccccccc}
1 & 1 & 0 & 0 & 0 & 0 & 0 & 0 & 0 & 0 & 0 & 0 & 0 & 0 & 0 & 0 \\
2 & 0 & 0 & 0 & 0 & 0 & 0 & 0 & 1 & 1 & 0 & 0 & 0 & 0 & 0 & 0
\end{array}\right)
$$

4. $a, d=1 \bmod 2, b=0 \bmod 3, c=0 \bmod 4$

$$
A=\frac{1}{2}\left(\begin{array}{llllllllllllllll}
1 & 1 & 0 & 0 & 0 & 0 & 0 & 0 & 0 & 0 & 0 & 0 & 0 & 0 & 0 & 0 \\
0 & 0 & 0 & 0 & 0 & 0 & 0 & 0 & 0 & 0 & 0 & 0 & 0 & 0 & 0 & 0
\end{array}\right)
$$

For the $U$ modulus there are 4 modular groups:

1. $\frac{1}{3} a+\frac{2}{3} b-\frac{2}{3} c-\frac{1}{3} d \in Z, \frac{2}{3} a+\frac{1}{3} b-\frac{1}{3} c-\frac{2}{3} d \in Z$

$$
A=\mathbf{0}
$$

2. $\frac{1}{3} a+\frac{2}{3} b-\frac{2}{3} c-\frac{1}{3} d \in Z, \frac{2}{3} a+\frac{1}{3} b-\frac{1}{3} c-\frac{2}{3} d \in Z, a=1 \bmod 2, b=0 \bmod 2$, $-\frac{1}{3} a-\frac{1}{6} b+\frac{2}{3} c+\frac{1}{3} d \in Z, \frac{1}{6} a+\frac{1}{3} b-\frac{1}{3} c-\frac{1}{6} d \in Z,-\frac{1}{2}+\frac{2}{3} a+\frac{1}{3} b-\frac{1}{3} c-\frac{1}{6} d \in Z$

$$
A=\frac{1}{2}\left(\begin{array}{llllllllllllllll}
0 & 0 & 0 & 0 & 0 & 0 & 0 & 0 & 0 & 0 & 0 & 0 & 0 & 0 & 0 & 0 \\
2 & 0 & 0 & 0 & 0 & 0 & 0 & 0 & 0 & 0 & 0 & 0 & 0 & 0 & 0 & 0
\end{array}\right)
$$

3. $\frac{1}{3} a+\frac{2}{3} b-\frac{2}{3} c-\frac{1}{3} d \in Z, \frac{2}{3} a+\frac{1}{3} b-\frac{1}{3} c-\frac{2}{3} d \in Z, a=1 \bmod 2, b=0 \bmod 2, d=$ $1 \bmod 2, c=0 \bmod 2,-\frac{1}{2}-\frac{1}{3} a-\frac{5}{12} b+\frac{11}{12} c+\frac{5}{6} d \in Z,-\frac{1}{2}-\frac{1}{4} a-\frac{1}{2} b+\frac{1}{2} c+\frac{3}{4} d \in Z$, 
$-\frac{1}{2}-\frac{11}{12} a+\frac{5}{6} b-\frac{5}{6} c-\frac{5}{12} d \in Z,-\frac{1}{2}+\frac{1}{2} a-\frac{1}{4} b-\frac{1}{4} c+\in Z, \frac{1}{2}+\frac{1}{6} a+\frac{1}{3} b-\frac{1}{3} c-\frac{2}{3} d \in Z$,
$\frac{1}{3} a-\frac{2}{3} b+\frac{1}{6} c+\frac{1}{3} d \in Z$,
$\quad A=\frac{1}{2}\left(\begin{array}{cccccccccccccccc}1 & 1 & 0 & 0 & 0 & 0 & 0 & 0 & 0 & 0 & 0 & 0 & 0 & 0 & 0 & 0 \\ 2 & 0 & 0 & 0 & 0 & 0 & 0 & 0 & 1 & 1 & 0 & 0 & 0 & 0 & 0 & 0\end{array}\right)$

4. $\frac{1}{3} a+\frac{2}{3} b-\frac{2}{3} c-\frac{1}{3} d \in Z, \frac{2}{3} a+\frac{1}{3} b-\frac{1}{3} c-\frac{2}{3} d \in Z, c=0 \bmod 2, d=1 \bmod 2$, $\frac{1}{3} a+\frac{2}{3} b-\frac{1}{6} c-\frac{1}{3} d \in Z, \frac{1}{2}+\frac{1}{6} a+\frac{1}{3} b-\frac{1}{3} c-\frac{2}{3} d \in Z$,

$$
A=\frac{1}{2}\left(\begin{array}{llllllllllllllll}
1 & 1 & 0 & 0 & 0 & 0 & 0 & 0 & 0 & 0 & 0 & 0 & 0 & 0 & 0 & 0 \\
0 & 0 & 0 & 0 & 0 & 0 & 0 & 0 & 0 & 0 & 0 & 0 & 0 & 0 & 0 & 0
\end{array}\right)
$$

\section{E.5 $\quad Z_{6}-\mathbf{I I}-\mathbf{c} S U(3) \times S O(7) \times S U(2)$}

There is a non- $T_{2} \oplus T_{4} Z_{2}$ fixed plane in the $\theta^{2}$ twisted sector.

$R=\left(\begin{array}{ll}1 & 0 \\ 0 & 1\end{array}\right) \quad K=\left(\begin{array}{ll}3 & 0 \\ 0 & 1\end{array}\right)$

Wilson lines as stated here correspond to thes six component line as: $A=$ $\left(\begin{array}{c}A_{5} \\ A_{6}\end{array}\right)$

For the $T$ modulus there are 5 modular groups:

1. $\Gamma^{0}(3)$

$$
A=\mathbf{0}
$$

2. $a=1 \bmod 4, b=0 \bmod 3, c=0 \bmod 4, d=1 \bmod 4$ and $a=3 \bmod 4$, $b=0 \bmod 3, c=0 \bmod 4, d=3 \bmod 4$

$$
A=\frac{1}{2}\left(\begin{array}{cccccccccccccccc}
0 & 0 & 0 & 0 & 0 & 0 & 0 & 0 & 0 & 0 & 0 & 0 & 0 & 0 & 0 & 0 \\
1 & 1 & 0 & 0 & 0 & 0 & 0 & 0 & 0 & 0 & 0 & 0 & 0 & 0 & 0 & 0
\end{array}\right)
$$

3. $a, d=1 \bmod 2, b=0 \bmod 3, c=0 \bmod 2$

$$
A=\frac{1}{2}\left(\begin{array}{cccccccccccccccc}
0 & 0 & 0 & 0 & 0 & 0 & 0 & 0 & 0 & 0 & 0 & 0 & 0 & 0 & 0 & 0 \\
2 & 0 & 0 & 0 & 0 & 0 & 0 & 0 & 0 & 0 & 0 & 0 & 0 & 0 & 0 & 0
\end{array}\right)
$$

4. $a, d=1 \bmod 4, b=0 \bmod 3, c=0 \bmod 8$ and $a, d=3 \bmod 4, b=$ $0 \bmod 3, c=0 \bmod 8$ and

$$
A=\frac{1}{4}\left(\begin{array}{cccccccccccccccc}
2 & 2 & 0 & 0 & 0 & 0 & 0 & 0 & 2 & 2 & 0 & 0 & 0 & 0 & 0 & 0 \\
0 & 0 & 0 & 0 & 0 & 0 & 0 & 0 & 1 & -1 & 1 & -1 & -1 & 1 & 1 & -1
\end{array}\right)
$$

5. $a, d=1 \bmod 2, b=0 \bmod 3, c=0 \bmod 4$

$$
A=\frac{1}{2}\left(\begin{array}{cccccccccccccccc}
1 & 1 & 0 & 0 & 0 & 0 & 0 & 0 & 1 & 1 & 0 & 0 & 0 & 0 & 0 & 0 \\
0 & 0 & 0 & 0 & 0 & 0 & 0 & 0 & 0 & 0 & 2 & 0 & 0 & 0 & 0 & 0
\end{array}\right)
$$

For the $U$ modulus there are 7 modular groups:

1. $\Gamma_{0}(3)$

$$
A=\mathbf{0}
$$

2. $a, d=1 \bmod 4, c=0 \bmod 12$ and $a, d=3 \bmod 4, c=0 \bmod 12$

$$
A=\frac{1}{2}\left(\begin{array}{llllllllllllllll}
0 & 0 & 0 & 0 & 0 & 0 & 0 & 0 & 0 & 0 & 0 & 0 & 0 & 0 & 0 & 0 \\
1 & 1 & 0 & 0 & 0 & 0 & 0 & 0 & 0 & 0 & 0 & 0 & 0 & 0 & 0 & 0
\end{array}\right)
$$

3. $a, d=1 \bmod 2, c=0 \bmod 6$

$$
A=\frac{1}{2}\left(\begin{array}{cccccccccccccccc}
0 & 0 & 0 & 0 & 0 & 0 & 0 & 0 & 0 & 0 & 0 & 0 & 0 & 0 & 0 & 0 \\
2 & 0 & 0 & 0 & 0 & 0 & 0 & 0 & 0 & 0 & 0 & 0 & 0 & 0 & 0 & 0
\end{array}\right)
$$


4. $a=1 \bmod 2, b=0 \bmod 2, c=0 \bmod 3, d=1 \bmod 2$

$$
A=\frac{1}{2}\left(\begin{array}{llllllllllllllll}
1 & 1 & 0 & 0 & 0 & 0 & 0 & 0 & 1 & 1 & 0 & 0 & 0 & 0 & 0 & 0 \\
0 & 0 & 0 & 0 & 0 & 0 & 0 & 0 & 0 & 0 & 0 & 0 & 0 & 0 & 0 & 0
\end{array}\right)
$$

5. $a, d=1 \bmod 4, b=0 \bmod 2, c=0 \bmod 12$ and $a, d=3 \bmod 4, b=$ $0 \bmod 2, c=0 \bmod 12$

$$
A=\frac{1}{4}\left(\begin{array}{cccccccccccccccc}
2 & 2 & 0 & 0 & 0 & 0 & 0 & 0 & 2 & 2 & 0 & 0 & 0 & 0 & 0 & 0 \\
0 & 0 & 0 & 0 & 0 & 0 & 0 & 0 & 1 & -1 & 1 & -1 & -1 & 1 & 1 & -1
\end{array}\right)
$$

6. $a, d=1 \bmod 2, b=0 \bmod 2, c=0 \bmod 6$

$$
A=\frac{1}{2}\left(\begin{array}{cccccccccccccccc}
1 & 1 & 0 & 0 & 0 & 0 & 0 & 0 & 1 & 1 & 0 & 0 & 0 & 0 & 0 & 0 \\
0 & 0 & 0 & 0 & 0 & 0 & 0 & 0 & 0 & 0 & 2 & 0 & 0 & 0 & 0 & 0
\end{array}\right)
$$

7. $a, d=0 \bmod 2, b=1 \bmod 2, c=3 \bmod 6$ and $a, d=1 \bmod 2, b=$ $0 \bmod 2, c=0 \bmod 6$

$$
A=\frac{1}{2}\left(\begin{array}{cccccccccccccccc}
2 & 0 & 0 & 0 & 0 & 0 & 0 & 0 & 0 & 0 & 0 & 0 & 0 & 0 & 0 & 0 \\
0 & 2 & 0 & 0 & 0 & 0 & 0 & 0 & 0 & 0 & 0 & 0 & 0 & 0 & 0 & 0
\end{array}\right)
$$

\section{E.6 $\quad Z_{6}-\mathbf{I I}-\mathbf{f} S U(3) \times S U(4)^{[2]} \times S U(2)$}

There is a non- $T_{2} \oplus T_{4} Z_{2}$ fixed plane in the $\theta^{2}$ twisted sector.

$R=\left(\begin{array}{ll}1 & 0 \\ 0 & 1\end{array}\right) \quad K=\left(\begin{array}{ll}3 & 0 \\ 0 & 1\end{array}\right)$

Wilson lines as stated here correspond to thes six component line as: $A=$ $\left(\begin{array}{l}A_{3} \\ A_{6}\end{array}\right)$

For the $T$ modulus there are 2 modular groups:

1. $\Gamma^{0}(3)$

$$
A=\mathbf{0}
$$

2. $a, d=1 \bmod 2, b=0 \bmod 3, c=0 \bmod 2$

$$
A=\frac{1}{2}\left(\begin{array}{llllllllllllllll}
0 & 0 & 0 & 0 & 0 & 0 & 0 & 0 & 0 & 0 & 0 & 0 & 0 & 0 & 0 & 0 \\
2 & 0 & 0 & 0 & 0 & 0 & 0 & 0 & 2 & 0 & 0 & 0 & 0 & 0 & 0 & 0
\end{array}\right)
$$

For the $U$ modulus there are 4 modular groups:

\section{1. $\Gamma_{0}(3)$}

$$
A=\mathbf{0}
$$

2. $a, d=1 \bmod 2, c=0 \bmod 6$

$$
A=\frac{1}{2}\left(\begin{array}{cccccccccccccccc}
0 & 0 & 0 & 0 & 0 & 0 & 0 & 0 & 0 & 0 & 0 & 0 & 0 & 0 & 0 & 0 \\
2 & 0 & 0 & 0 & 0 & 0 & 0 & 0 & 2 & 0 & 0 & 0 & 0 & 0 & 0 & 0
\end{array}\right)
$$

3. $a, d=0 \bmod 2, b=1 \bmod 2, c=3 \bmod 6$ and $a, d=1 \bmod 2, b=$ $0 \bmod 2, c=0 \bmod 6$

$$
A=\frac{1}{2}\left(\begin{array}{cccccccccccccccc}
2 & 0 & 0 & 0 & 0 & 0 & 0 & 0 & 0 & 0 & 0 & 0 & 0 & 0 & 0 & 0 \\
2 & 0 & 0 & 0 & 0 & 0 & 0 & 0 & 0 & 0 & 0 & 0 & 0 & 0 & 0 & 0
\end{array}\right)
$$

4. $a=1 \bmod 2, b=0 \bmod 2, c=0 \bmod 3, d=1 \bmod 2$

$$
A=\frac{1}{2}\left(\begin{array}{cccccccccccccccc}
2 & 0 & 0 & 0 & 0 & 0 & 0 & 0 & 2 & 0 & 0 & 0 & 0 & 0 & 0 & 0 \\
0 & 0 & 0 & 0 & 0 & 0 & 0 & 0 & 0 & 0 & 0 & 0 & 0 & 0 & 0 & 0
\end{array}\right)
$$




\section{E.7 $\quad Z_{6}-\mathbf{I I}-\mathbf{g} S U(3) \times S p(6) \times S U(2)$}

There is a non- $T_{2} \oplus T_{4} Z_{2}$ fixed plane in the $\theta^{2}$ twisted sector.

$R=\left(\begin{array}{ll}1 & 0 \\ 0 & 1\end{array}\right) \quad K=\left(\begin{array}{ll}3 & 0 \\ 0 & 1\end{array}\right)$

Wilson lines as stated here correspond to thes six component line as: $A=$ $\left(\begin{array}{c}A_{5} \\ A_{6}\end{array}\right)$

For the $T$ modulus there are 2 modular groups:

1. $\Gamma^{0}(3)$

$$
A=\mathbf{0}
$$

2. $a, d=1 \bmod 2, b=0 \bmod 3, c=0 \bmod 2$

$$
A=\frac{1}{2}\left(\begin{array}{cccccccccccccccc}
0 & 0 & 0 & 0 & 0 & 0 & 0 & 0 & 0 & 0 & 0 & 0 & 0 & 0 & 0 & 0 \\
2 & 0 & 0 & 0 & 0 & 0 & 0 & 0 & 2 & 0 & 0 & 0 & 0 & 0 & 0 & 0
\end{array}\right)
$$

For the $U$ modulus there are 4 modular groups:

1. $\Gamma_{0}(3)$

$$
A=\mathbf{0}
$$

2. $a, d=1 \bmod 2, c=0 \bmod 6$

$$
A=\frac{1}{2}\left(\begin{array}{llllllllllllllll}
0 & 0 & 0 & 0 & 0 & 0 & 0 & 0 & 0 & 0 & 0 & 0 & 0 & 0 & 0 & 0 \\
2 & 0 & 0 & 0 & 0 & 0 & 0 & 0 & 2 & 0 & 0 & 0 & 0 & 0 & 0 & 0
\end{array}\right)
$$

3. $a, d=0 \bmod 2, b=1 \bmod 2, c=3 \bmod 6$ and $a, d=1 \bmod 2, b=$ $0 \bmod 2, c=0 \bmod 6$

$$
A=\frac{1}{2}\left(\begin{array}{cccccccccccccccc}
2 & 0 & 0 & 0 & 0 & 0 & 0 & 0 & 0 & 0 & 0 & 0 & 0 & 0 & 0 & 0 \\
2 & 0 & 0 & 0 & 0 & 0 & 0 & 0 & 0 & 0 & 0 & 0 & 0 & 0 & 0 & 0
\end{array}\right)
$$

4. $a=1 \bmod 2, b=0 \bmod 2, c=0 \bmod 3, d=1 \bmod 2$

$$
A=\frac{1}{2}\left(\begin{array}{cccccccccccccccc}
2 & 0 & 0 & 0 & 0 & 0 & 0 & 0 & 2 & 0 & 0 & 0 & 0 & 0 & 0 & 0 \\
0 & 0 & 0 & 0 & 0 & 0 & 0 & 0 & 0 & 0 & 0 & 0 & 0 & 0 & 0 & 0
\end{array}\right)
$$

\section{E.8 $Z_{8}-\mathbf{I I}-\mathbf{a} S O(10) \times S U(2)$}

There is a non- $T_{2} \oplus T_{4} Z_{2}$ fixed plane in the $\theta^{2}$ twisted sector.

$R=\left(\begin{array}{ll}2 & 0 \\ 0 & 1\end{array}\right) \quad K=\left(\begin{array}{ll}2 & 0 \\ 0 & 1\end{array}\right)$

Wilson lines as stated here correspond to thes six component line as: $A=$ $\left(\begin{array}{l}A_{4} \\ A_{6}\end{array}\right)$

For the $T$ modulus there are 6 modular groups:

1. $\Gamma^{0}(2)$

$$
A=\mathbf{0}
$$

2. $a, d=1 \bmod 4, b=0 \bmod 2, c=0 \bmod 4$ and $a, d=3 \bmod 4, b=$ $0 \bmod 2, c=0 \bmod 4$

$$
A=\frac{1}{2}\left(\begin{array}{cccccccccccccccc}
0 & 0 & 0 & 0 & 0 & 0 & 0 & 0 & 0 & 0 & 0 & 0 & 0 & 0 & 0 & 0 \\
1 & 1 & 0 & 0 & 0 & 0 & 0 & 0 & 0 & 0 & 0 & 0 & 0 & 0 & 0 & 0
\end{array}\right)
$$

3. $a, d=1 \bmod 2, b=0 \bmod 2, c=0 \bmod 2$ 


$$
A=\frac{1}{2}\left(\begin{array}{cccccccccccccccc}
0 & 0 & 0 & 0 & 0 & 0 & 0 & 0 & 0 & 0 & 0 & 0 & 0 & 0 & 0 & 0 \\
2 & 0 & 0 & 0 & 0 & 0 & 0 & 0 & 0 & 0 & 0 & 0 & 0 & 0 & 0 & 0
\end{array}\right)
$$

4. $a=1 \bmod 4, b=0 \bmod 2, c=0 \bmod 2, d=1 \bmod 2$

$$
A=\frac{1}{4}\left(\begin{array}{cccccccccccccccc}
3 & 2 & 1 & 1 & 1 & 0 & 0 & 0 & 4 & 0 & 0 & 0 & 0 & 0 & 0 & 0 \\
0 & 0 & 0 & 0 & 0 & 0 & 0 & 0 & 0 & 0 & 0 & 0 & 0 & 0 & 0 & 0
\end{array}\right)
$$

5. $a=1 \bmod 4, b=0 \bmod 2, c=0 \bmod 8, d=1 \bmod 4$

$$
A=\frac{1}{4}\left(\begin{array}{cccccccccccccccc}
3 & 2 & 1 & 1 & 1 & 0 & 0 & 0 & 4 & 0 & 0 & 0 & 0 & 0 & 0 & 0 \\
0 & 0 & 0 & 0 & 0 & 0 & 0 & 0 & 0 & 0 & 0 & 0 & 0 & 0 & 0 & 0
\end{array}\right)
$$

6. $a=1 \bmod 4, b=0 \bmod 2, c=0 \bmod 4, d=1 \bmod 2$

$$
A=\frac{1}{4}\left(\begin{array}{llllllllllllllll}
3 & 2 & 1 & 1 & 1 & 0 & 0 & 0 & 4 & 0 & 0 & 0 & 0 & 0 & 0 & 0 \\
0 & 0 & 0 & 0 & 0 & 0 & 0 & 0 & 0 & 2 & 2 & 0 & 0 & 0 & 0 & 0
\end{array}\right)
$$

For the $U$ modulus there are 6 modular groups:

1. $\Gamma_{0}(2)$

$$
A=\mathbf{0}
$$

2. $a, d=1 \bmod 4, c=0 \bmod 8$ and $a, d=3 \bmod 4, c=0 \bmod 8$

$$
A=\frac{1}{2}\left(\begin{array}{cccccccccccccccc}
0 & 0 & 0 & 0 & 0 & 0 & 0 & 0 & 0 & 0 & 0 & 0 & 0 & 0 & 0 & 0 \\
1 & 1 & 0 & 0 & 0 & 0 & 0 & 0 & 0 & 0 & 0 & 0 & 0 & 0 & 0 & 0
\end{array}\right)
$$

3. $a, d=1 \bmod 2, c=0 \bmod 4$

$$
A=\frac{1}{2}\left(\begin{array}{cccccccccccccccc}
0 & 0 & 0 & 0 & 0 & 0 & 0 & 0 & 0 & 0 & 0 & 0 & 0 & 0 & 0 & 0 \\
2 & 0 & 0 & 0 & 0 & 0 & 0 & 0 & 0 & 0 & 0 & 0 & 0 & 0 & 0 & 0
\end{array}\right)
$$

4. $a=1 \bmod 2, b=0 \bmod 2, c=0 \bmod 2, d=1 \bmod 4$

$$
A=\frac{1}{4}\left(\begin{array}{cccccccccccccccc}
3 & 2 & 1 & 1 & 1 & 0 & 0 & 0 & 4 & 0 & 0 & 0 & 0 & 0 & 0 & 0 \\
0 & 0 & 0 & 0 & 0 & 0 & 0 & 0 & 0 & 0 & 0 & 0 & 0 & 0 & 0 & 0
\end{array}\right)
$$

5. $a=1 \bmod 4, b=0 \bmod 2, c=0 \bmod 8, d=1 \bmod 4$

$$
A=\frac{1}{4}\left(\begin{array}{llllllllllllllll}
3 & 2 & 1 & 1 & 1 & 0 & 0 & 0 & 4 & 0 & 0 & 0 & 0 & 0 & 0 & 0 \\
0 & 0 & 0 & 0 & 0 & 0 & 0 & 0 & 0 & 0 & 0 & 0 & 0 & 0 & 0 & 0
\end{array}\right)
$$

6. $a=1 \bmod 2, b=0 \bmod 2, c=0 \bmod 4, d=1 \bmod 4$

$$
A=\frac{1}{4}\left(\begin{array}{llllllllllllllll}
3 & 2 & 1 & 1 & 1 & 0 & 0 & 0 & 4 & 0 & 0 & 0 & 0 & 0 & 0 & 0 \\
0 & 0 & 0 & 0 & 0 & 0 & 0 & 0 & 0 & 2 & 2 & 0 & 0 & 0 & 0 & 0
\end{array}\right)
$$

\section{E.9 $Z_{12}-\mathbf{I}-\mathbf{a} E_{6}$}

There is a non- $T_{2} \oplus T_{4} Z_{3}$ fixed plane in the $\theta^{3}$ twisted sector.

$R=\left(\begin{array}{ll}2 & 0 \\ 0 & 2\end{array}\right) \quad K=\left(\begin{array}{ll}2 & 0 \\ 0 & 2\end{array}\right)$

Wilson lines as stated here correspond to thes six component line as: $A=$ $\left(\begin{array}{l}A_{1} \\ A_{4}\end{array}\right)$

For the $T$ modulus there are 2 modular groups:

1. $\Gamma^{0}(2)$

$$
A=\mathbf{0}
$$

2. $a, d=1 \bmod 3, b=0 \bmod 2, c=0 \bmod 3$

$$
A=\frac{1}{3}\left(\begin{array}{llllllllllllllll}
2 & 0 & 0 & 0 & 0 & 0 & 0 & 0 & 1 & 1 & 0 & 0 & 0 & 0 & 0 & 0 \\
2 & 0 & 0 & 0 & 0 & 0 & 0 & 0 & 1 & 1 & 0 & 0 & 0 & 0 & 0 & 0
\end{array}\right)
$$




\section{F Calculation of $\mathbf{R}$ and $\mathrm{K}$ matrices}

The matrices $R$ and $K$ allow the constraints on the modular symmetry group of non- $T_{2} \oplus T_{4}$ orbifolds to be expressed in a 2-dimensional form. The constraints are obtained by ensuring that the physical winding and momentum states in the fixed plane transform properly under the modular transformations. The physical winding numbers and momenta in the plane must be expressable in terms of only two independent components in each case. In particular we wish to express the contribution to the worldsheet momentum $P$ in terms of the two independent values of the momenta and windings, hence $K$ is defined by

$$
P=p_{\text {soln }}^{T} w_{\text {soln }}=m_{\perp}^{T} K^{T} n_{\perp}
$$

where $p_{\text {soln }}$ and $w_{\text {soln }}$ are the solutions to $\left(Q^{*}\right)^{k} p=p$ and $Q^{k} w=w$ respectively and are six dimensional vectors and $m_{\perp}, n_{\perp}$ are the corresponding two dimensional momentum and winding numbers. An example of the calculation of $K$ for a specific orbifold was given in appendix C. Similarly, the $R$ matrix is introduced when expressing the constraints in the presence of non-zero Wilson lines in terms of only two independent lines. The Wilson lines involved in the problem are those which correspond to the windings of $w_{\text {soln }}$. For example, in the case of the $Z_{6}-\mathrm{II}-\mathrm{f}$ of appendix $\mathrm{C}, w_{\text {soln }}$ is given by

$w_{\text {soln }}=\left(n_{1}, 2 n_{1},-n_{1}, 0,0, n_{6}\right)^{T}$, and so the Wilson lines involved in the problem are the components $A_{1}, A_{2}, A_{3}$ associated with windings in one direction in the fixed plane and $A_{6}$ associated with the windings in the other direction. It might appear that there are then in fact 4 different Wilson line components involved in the problem, but the number of independent Wilson line components is reduced to only two in all problems by the point group invariance condition, which in this case yields the relations $A_{2} \approx A_{3}, A_{1} \approx 2 A_{2} \approx 0$. So in the case of the $Z_{6}$-II-f orbifold the Wilson line contribution can be expressed in terms of the independent components $A_{2}$ and $A_{6}$. The $R$ matrix is then defined by

$$
A w_{\text {soln }}=A_{\perp} R n_{\perp}
$$

where $A$ is the six dimensional Wilson line and $A_{\perp}$ is the two dimensional line consisting of the two independent components of the six dimensional line which contribute, as discussed above, and $n_{\perp}$ is as defined above.

\section{References}


[1] L. Dixon, J.A. Harvey, C.Vafa and E.Witten, Nucl. Phys. B261 (1985) 678; B274(1986) 285.

[2] A. Font, L.E. Ibáñez, F. Quevedo and A. Sierra, Nucl. Phys. B331 (1991) 421.

[3] R. Dijkgraaf, E.Verlinde and H.Verlinde, Comm. Math. Phys. 115 (1988) 649 .

[4] R. Dijkgraaf, E.Verlinde and H.Verlinde, On Moduli Spaces of Conformal Field Theories with $c \geq 1$, Proceedings Copenhagen Conference, Perspectives in String Theory, edited by P. Di Vecchia and J.L. Petersen, World Scientific, Singapore, 1988.

[5] A. Shapere and F.Wilczek, Nucl. Phys. B320 (1989) 669.

[6] M. Dine, P. Huet and M.Seiberg, Nucl. Phys. B322 (1989) 301.

[7] J. Lauer, J.Mas and H.P. Nilles, Nucl. Phys. B351 (1991) 353.

[8] K. Kikkawa and M. Yamasaki, Phys. Lett. B149, (1984) 357; N.Sakai and I.Senda, Prog. Theor. Phys. 75 (1984) 692.

[9] A. Giveon, E. Rabinovici and G.Veneziano, Nucl. Phys. B322 (1989) 167.

[10] W. Lerche, D. Lüst and N.P. Warner, Phys. Lett. B231 (1989) 417.

[11] V.S. Kaplunovsky, Nucl. Phys. B307 (1988) 145.

[12] L.J. Dixon, V.S. Kaplunovsky and J. Louis, Nucl. Phys. B355 (1991) 649.

[13] L.E. Ibáñez, D. Lüst and G.G. Ross, Phys. Lett. B272 (1991) 25.

[14] L.E. Ibáñez and D. Lüst, Nucl. Phys. B382 (1992) 305.

[15] J.P. Deredinger, S.Ferrara, C.Kounas and F.Zwirner, Nucl. Phys. B372 (1992) 145, Phys. Lett. B271 (1991) 307.

[16] D. Bailin and A. Love, Phys. Lett. B278 (1992) 125; Phys. Lett. B292 (1992) 315.

[17] A. Font, L.E. Ibáñez, D. Lüst and F. Quevedo, Phys. Lett.B245 (1990) 401; S. Ferrara, N. Magnoli, T.R. Taylor and G. Veneziano, ibid. B245 (1990) 409; M.Cvetic, A.Font, L.E. Ibáñez, D. Lüst and F. Quevedo, Nucl. Phys. B361 (1991) 194. 
[18] L. Dixon, talk presented st the APSDPF. Meeting at Houston (1990); V. Kaplunovsky, talk presented at Strings 90 workshop at College Station (World Scientific, 1991); L. Dixon, V. Kaplunovsky, J. Louis and M. Peskin, unpublished.

[19] J.A. Casas, Z. Lalak, C. Munoz and G.G. Ross, Nucl. Phys. B347 (1990) 243.

[20] H.P. Nilles and M. Olechowsky, Phys. Lett. B248 (1990) 268; P. Binetray and M.K. Gaillard, ibid. B253 (1991) 119.

[21] J. Louis, SLAC-PUB-5645 (1991)

[22] D. Lüst and T.R. Taylor, Phys. Lett. B253 (1991) 335.

[23] D. Lüst and C. Munoz, Phys. Lett. B279 (1992) 272; B. de Carlos, J.A. Casas and C. Munoz, Nucl. Phys. B339 (1993) 623

[24] P. Mayr and S. Stieberger, Nucl. Phys. B407 (1993) 725.

[25] D. Bailin, A. Love, W. Sabra and S. Thomas, Phys. Lett. B320 (1994) 21.

[26] D. Bailin, A. Love, W. Sabra and S. Thomas, Mod. Phys. Lett. A9 (1994) 67.

[27] M. Spalinski, Phys. Lett. B275 (1992) 47; J. Erler, D. Jungnickel and H.P. Nilles, ibid. B276 (1992) 303.

[28] J. Erler and M. Spalinski, MPI-PH-92-61 (1992).

[29] D. Bailin, A. Love, W. Sabra and S. Thomas, Mod. Phys. Lett. A9 (1994) 1229.

[30] A. Love, W. Sabra and S. Thomas, Nucl. Phys. B427 (1994) 181.

[31] Y. Katsuki, Y. Kawamura, T. Kobayashi, N. Ohtsubo, Y. Ono and K. Tanioka, Nucl. Phys. B341 (1990) 611.

[32] T. Kobayashi and N. Ohtsubo, Phys. Lett. B262 (1991) 425.

[33] D. Bailin, A. Love and W. Sabra, Nucl. Phys. B403 (1993) 265.

[34] T. Kobayashi and N. Ohtsubo, Int. J. Mod. Phys. A9 (1994) 87.

[35] A. Font, L.E. Ibáñez, F. Quevedo and A. Sierra, Nucl. Phys. B331 (1990) 421. 
[36] L.E. Ibáñez, J. Mas, H.P. Nilles and F.Quevedo, Nucl. Phys. B301 (1988) 157.

[37] D. Bailin, A. Love, W. Sabra and S. Thomas, Mod. Phys. Lett. A10 (1995) 337.

[38] S. Todd, Ph.D. Thesis 